\title{
The Nirspec Ultracool Dwarf Radial Velocity Survey
}

\section{Citation}

Blake, Cullen H., David Charbonneau, and Russel J. White. 2010. "THE NIRSPEC ULTRACOOL DWARF RADIAL VELOCITY SURVEY." The Astrophysical Journal 723 (1): 684-706. https:// doi.org/10.1088/0004-637x/723/1/684.

\section{Permanent link}

http://nrs.harvard.edu/urn-3:HUL.InstRepos:41397417

\section{Terms of Use}

This article was downloaded from Harvard University's DASH repository, and is made available under the terms and conditions applicable to Other Posted Material, as set forth at http:// nrs.harvard.edu/urn-3:HUL.InstRepos:dash.current.terms-of-use\#LAA

\section{Share Your Story}

The Harvard community has made this article openly available.

Please share how this access benefits you. Submit a story.

\section{Accessibility}




\title{
THE NIRSPEC ULTRACOOL DWARF RADIAL VELOCITY SURVEY
}

\author{
Cullen H. Blake ${ }^{1,2}$, David Charbonneau ${ }^{2}$, and Russel J. White ${ }^{3}$ \\ ${ }^{1}$ Department of Astrophysical Sciences, Princeton University, Peyton Hall, Ivy Lane, Princeton, NJ 08544, USA \\ ${ }^{2}$ Harvard-Smithsonian Center for Astrophysics, 60 Garden Street, Cambridge, MA 02138, USA \\ ${ }^{3}$ Department of Physics and Astronomy, Georgia State University, Atlanta, GA 30303, USA \\ Received 2010 March 13; accepted 2010 August 23; published 2010 October 13
}

\begin{abstract}
We report the results of an infrared Doppler survey designed to detect brown dwarf and giant planetary companions to a magnitude-limited sample of ultracool dwarfs. Using the NIRSPEC spectrograph on the Keck II telescope, we obtained approximately 600 radial velocity (RV) measurements over a period of six years of a sample of 59 late-M and L dwarfs spanning spectral types M8/L0 to L6. A subsample of 46 of our targets has been observed on three or more epochs. We rely on telluric $\mathrm{CH}_{4}$ absorption features in Earth's atmosphere as a simultaneous wavelength reference and exploit the rich set of $\mathrm{CO}$ absorption features found in the $K$-band spectra of cool stars and brown dwarfs to measure RVs and projected rotational velocities. For a bright, slowly rotating $\mathrm{M}$ dwarf standard we demonstrate an RV precision of $50 \mathrm{~m} \mathrm{~s}^{-1}$ and for slowly rotating L dwarfs we achieve a typical RV precision of approximately $200 \mathrm{~m} \mathrm{~s}^{-1}$. This precision is sufficient for the detection of close-in giant planetary companions to midL dwarfs as well as more equal mass spectroscopic binary systems with small separations $(a<2$ AU). We present an orbital solution for the subdwarf binary LSR1610-0040 as well as an improved solution for the M/T binary 2M0320-04. We compare the distribution of our observed values for the projected rotational velocities, $V$ sin $i$, to those in the literature and find that our sample contains examples of slowly rotating mid-L dwarfs, which have not been seen in other surveys. We also combine our RV measurements with distance estimates and proper motions from the literature and estimate the dispersion of the space velocities of the objects in our sample. Using a kinematic age estimate, we conclude that our UCDs have an age of $5.0_{-0.6}^{+0.7} \mathrm{Gyr}$, similar to that of nearby sun-like stars. We simulate the efficiency with which we detect spectroscopic binaries and find that the rate of tight $(a<1$ AU) binaries in our sample is $2.5_{-1.6}^{+8.6} \%$, consistent with recent estimates in the literature of a tight binary fraction of $3 \%-4 \%$.
\end{abstract}

Key words: brown dwarfs - stars: low-mass - techniques: radial velocities

Online-only material: machine-readable table

\section{INTRODUCTION}

Since the discovery of the prototypical "hot Jupiter" orbiting the star 51 Pegasi in 1995, more than 450 extrasolar planets have been identified. This flurry of discovery has been driven largely by technological innovation and the development of new observational techniques. Precise Doppler measurements have played a particularly important role in the discovery of extrasolar planets. The search for unseen orbiting companions to stars by measuring the subtle Doppler shifts of stellar spectral lines is more than a century old (e.g., Vogel 1901), and since the late 1800 s the precision of these measurements has improved by more than four orders of magnitude. Since the amplitude of the Doppler signal induced by an unseen companion is directly proportional to the companion's mass, the discovery of extrasolar planets is a direct result of steady improvements to measurement techniques that have been used for over a century to study binary star systems.

Approximately $4 \%$ of the known extrasolar planetary systems have low-mass star hosts (M0-M4; http://www.exoplanet.eu). The bias in Doppler surveys toward stars more massive than $M$ dwarfs is due largely to the technical limitations of obtaining precise measurements of cool, intrinsically faint objects. Understanding the rate of occurrence of planets orbiting these lowest mass stars and brown dwarfs, collectively referred to as ultracool dwarfs (UCDs; spectral types later than M5), may have important implications for theories of planet formation since the core accretion and disk instability formation scenarios make different predictions about the occurrence of planetary compan- ions as a function of host mass (Boss 2006). While it has been shown that early $M$ dwarfs have a relative paucity of close in Jupiter-mass planets (Endl et al. 2006; Johnson et al. 2007), we are just now beginning to understand the occurrence of superEarth $\left(M_{p}<10 M_{\oplus}\right)$ planets to mid-M stars or any type of planet orbiting late-M or L dwarfs. Doppler planet surveys generally include no UCD targets (Bailey et al. 2009), so little is known about the rate at which planetary companions accompany the lowest mass stars and brown dwarfs. There are some initial indications from microlensing surveys that sub-Jupitermass companions orbiting at several AU from $\mathrm{M}$ dwarfs may be common (Gould et al. 2006b), but these findings are not yet statistically robust. Several examples of possible planetary companions to brown dwarfs do exist, including a 16-20 $M_{\mathrm{J}}$ companion found orbiting a young brown dwarf in a Doppler survey conducted by Joergens \& Müller (2007), a giant planetary companion to 2M1207-39 found by Chauvin et al. (2005) in a direct imaging survey, and a possible super-Earth orbiting the UCD MOA-2007-BLG-192 detected via microlensing (Bennett et al. 2008).

There is strong observational evidence for the initial stages of planet formation around young UCDs in the form of a high disk fraction (Luhman et al. 2008) and the formation of silicate grains (Apai et al. 2005). The formation of planets around UCDs with $M_{*}<0.1 M_{\odot}$ has been modeled by Payne \& Lodato (2007) who found that, depending on the mass of the protoplanetary disk, super-Earths up to $5 M_{\oplus}$ may be relatively common $(10 \%$ of UCDs) if the disk mass scales linearly with the host mass. The interaction of a planet with a gaseous disk leads to gravitational 
torques that can cause a planet to lose angular momentum and migrate within the disk. For small planets $\left(M_{p} \ll M_{\mathrm{J}}\right)$, the disk-planet interactions are linear, resulting in a Type I migration that can cause very rapid inward movement. More massive planets may be able to open a gap in the protoplanetary disk and then torques between the planet and the inner and outer edges of the gap may cause the planet to slowly migrate inward through Type II migration. UCD planets may be found at relatively large separations ( $a>1 \mathrm{AU}$ ) since inward migration to short orbital periods through Type II migration is not expected to be efficient in UCD protoplanetary disks. The detection of a significant population of close-in companions to UCDs would place interesting constraints on planetary migration via the Type I mechanism since the rapid inward movements due to this mechanism are expected to cause protoplanets to fall into the star on short timescales. Payne \& Lodato (2007) predict that the formation of giant planetary companions to UCDs should be completely inhibited and propose that systems like 2M1207-39 form in a manner similar to that of binary stars.

Doppler planet searches are very sensitive to spectroscopic binary systems. Binary star systems afford one of the few opportunities to directly measure the masses and radii of stars and measurements of stars in binary systems are an important component of the observational basis of our theoretical models of stellar structure and evolution. There are four different types of spectroscopic binary systems: single-lined (SB1), double-lined (SB2), single-lined eclipsing (SEB), and double-lined eclipsing (DEB). In an SB1 binary the reflex motion of the primary (more luminous and usually more massive) star is observed and when combined with the orbital period and eccentricity can be used to define a mass function, a transcendental equation involving the masses of both components and the inclination. The inclination of the system is not known, so additional observations, such as measurements of the astrometric orbit, are required to determine the actual masses of both components. In an SB2 system the spectral lines of both the primary and secondary are observed and the ratio of the radial velocity (RV) semi-amplitudes of the components directly determines the ratio of their masses ( $\left.q=M_{2} / M_{1}=K_{1} / K_{2}\right)$. Using a technique like TODCOR (Mazeh \& Zucker 1994) to analyze observations of an SB2 system it may also be possible to simultaneously determine mass and flux ratios of the components of the system, enabling direct tests of theoretical models of coeval low-mass stars and brown dwarfs. As with an SB1 system, the system inclination is not known in an SB2, so individual masses cannot be directly measured. In an SEB system, light from the secondary is not detected, but its presence is inferred from both the reflex motion of the primary and the diminution in brightness that occurs as the secondary eclipses the primary. In these systems, of which the transiting extrasolar planets are a specific case, the orbital inclination is constrained by the fact that an eclipse occurs. The DEB is a rare, but very important, type of binary that allows for precise measurements of the masses and radii of both components of the binary.

Very few UCD spectroscopic binaries are known, meaning that theoretical models of low-mass stars and brown dwarfs are relatively untested compared to models of sun-like stars. In fact, there are significant (5\%-10\%) discrepancies between models and mass and radius measurements of low-mass stars in eclipsing binary systems (Chabrier et al. 2007). For UCDs, only one DEB system is known (Stassun et al. 2006), serving as the lone observational benchmark for models of young brown dwarfs. In this young $(t<0.003 \mathrm{Gyr})$ system, the measured radii are consistent with theoretical models but the estimated temperatures indicate that the less massive component is actually hotter, contrary to theoretical predictions for coeval brown dwarfs. Since UCDs that appear brightest in the sky are necessarily close to the Sun, direct imaging surveys have been very successful in detecting UCD binaries with orbital separations $a>1 \mathrm{AU}$. The orbital motions in these long-period visual binary systems can be observed (e.g., Bouy et al. 2004; Dupuy et al. 2009; Martinache et al. 2009; Konopacky et al. 2010 ), providing another opportunity to measure masses of UCDs. Discovering additional UCD binaries, particularly SB2 and DEB systems, is crucial for improving our understanding of stellar astrophysics at and below the bottom of the main sequence.

Observations of a large sample of binary systems also enable tests of models of the formation history of UCDs. The process through which UCDs form is not well understood, and the statistical properties of the orbital separations and mass ratios of binary systems help to test potential formation scenarios. For example, some models of UCD formation suggest that these objects undergo ejection from star formation regions before they have the opportunity to accrete enough mass to become main-sequence stars (Whitworth at al. 2007; Luhman at al. 2006). If this is the case, wide binaries $(a>15 \mathrm{AU})$ with low binding energies are expected to be rare. Thanks to direct imaging surveys, the binary fraction at large separations has been well studied. Compared to Sun-like stars, UCD binaries tend to have larger mass ratios $(q \sim 1)$ and smaller separations $(a \sim 7 \mathrm{AU})$ and very few wide $(a>15 \mathrm{AU})$ systems are seen. Most systems with small $(a<1 \mathrm{AU})$ separations are not resolved by imaging surveys, but their relatively short periods (years instead of decades) make them prime targets for Doppler surveys. Since no comprehensive Doppler survey of UCDs has been carried out, our understanding of the overall distribution of system properties is incomplete at small separations (Burgasser at al. 2007; Allen 2007).

Obtaining precise Doppler measurements poses a significant technical challenge, particularly if the target is a UCD. In the 1970s, astronomers realized that having a simultaneous wavelength calibrator that superimposes absorption features of known wavelengths onto a source spectrum provides a major advantage in terms of Doppler precision for slit spectrographs (Griffin \& Griffin 1973). Prior to the advent of the gas cell technique (HF by Campbell 1983 and later $I_{2}$ ), atomic and molecular absorption features in Earth's atmosphere were used for this purpose. While not inherently stable like the gas in an absorption cell, telluric lines have been used to produce Doppler measurements with a month-to-month precision of 5-20 m s (Balthasar et al. 1982; Smith 1982; Caccin et al. 1985; Cochran 1988; Hatzes \& Cochran 1993; Figueira et al. 2010b). After making corrections based on a simple model of atmospheric winds, Figueira et al. (2010a) have demonstrated that atmospheric $\mathrm{O}_{2}$ lines at optical wavelengths can be used to make $\mathrm{RV}$ measurements with a precision of $2 \mathrm{~m} \mathrm{~s}^{-1}$ over timescales of years. Doppler measurements of Sun-like stars with a precision exceeding $1 \mathrm{~m} \mathrm{~s}^{-1}$ have been demonstrated using two different techniques for calibrating high-resolution spectroscopic data. For bright early-M dwarfs both the thorium-argon (ThAr) emission lamps and simultaneous iodine $\left(I_{2}\right)$ absorption cells have been used to obtain RV precision of 3-5 $\mathrm{m} \mathrm{s}^{-1}$ (Endl et al. 2006; Johnson et al. 2007; Udry et al. 2007; Zechmeister et al. 2009). Due to their cool temperatures (1200 $\mathrm{K}<T_{\text {eff }}<$ $2600 \mathrm{~K})$ and small sizes $\left(R_{*}=0.1 R_{\odot}\right)$, UCDs are intrinsi- 
cally very faint at the wavelengths where these measurements are made (400 to $700 \mathrm{~nm}$ ), limiting observations to only the few brightest targets on the sky. Making precise Doppler measurements at near-infrared (NIR) wavelengths is a very attractive option for exploring the population of planets orbiting UCDs.

Until quite recently, the precision of NIR Doppler measurements of UCDs has tended to lag behind that of RV measurements of Sun-like stars by two orders of magnitude. This is due in part to the relative complexity and expense of NIR echelle spectrographs, the relative faintness of cool dwarfs, and to the worse noise properties of NIR detectors compared to CCDs. In addition, there has been a lack of suitable wavelength references in the NIR. The $I_{2}$ cell is not effective at these wavelengths, but the Th lines in ThAr emission lamps may prove useful in $J$ and $H$ bands. Both Mahadevan \& Ge (2009) and Reiners et al. (2010) provide a summary of future prospects for calibrating NIR echelle spectra using gas absorption cells and emission line lamps. The NIR is replete with telluric absorption lines due to $\mathrm{H}_{2} \mathrm{O}$ and $\mathrm{CH}_{4}$. In this spectral region, UCDs have a rich set of molecular absorption features that can potentially be used to make precise Doppler measurements. We have developed a technique that relies on telluric $\mathrm{CH}_{4}$ absorption features as a simultaneous wavelength reference and exploits the rich set of $\mathrm{CO}$ absorption features found in the spectra of UCDs near $2.3 \mu \mathrm{m}$ to make Doppler measurements with a limiting precision of approximately $50 \mathrm{~m} \mathrm{~s}^{-1}$. High-resolution NIR spectrographs that could make use of this technique are expected to be an important component of the suites of instruments on future large telescopes, and Ramsey et al. (2008) and Erskine et al. (2005) report the development of new high-resolution instruments that should be able to achieve $10 \mathrm{~m} \mathrm{~s}^{-1}$ precision at NIR wavelengths. Doppler measurements with a precision of $\sigma_{\mathrm{RV}}<300 \mathrm{~m} \mathrm{~s}^{-1}$ have been demonstrated by Martín et al. (2006), Blake et al. (2007), Blake et al. (2008a), Prato et al. (2008), and Zapatero Osorio et al. (2009), and a Doppler precision in the range 5-20 m s${ }^{-1}$ has been demonstrated on short timescales using the CRIRES instrument on the Very Large Telescope (VLT) by Huélamo et al. (2008), Seifahrt \& Käufl (2008), and Figueira et al. (2010b). Recently, Bean et al. (2010) used an $\mathrm{NH}_{3}$ absorption cell with CRIRES to obtain RV measurements of bright $(K<8)$ mid-M dwarfs in the $K$ band with a precision approaching $5 \mathrm{~m} \mathrm{~s}^{-1}$. We do not expect to reach this impressive level of precision for two reasons: first, the resolution of NIRSPEC is $R=25,000$, which is significantly worse than that of CRIRES $(R=100,000)$. Second, all but 8 of the 59 targets in our survey have $K$ magnitudes between 11 and 12.5 , significantly fainter than the faintest targets reported in Bean et al. (2010) or Figueira et al. (2010b).

We report the results of a Doppler survey of 59 UCDs using the NIRSPEC instrument on the Keck II telescope. Our observations span a period of six years and we demonstrate sensitivity to giant planetary companions as well as UCD-UCD binaries with small orbital separations. In Section 2, we describe our UCD sample and our NIRSPEC observations. In Section 3, we describe the details of our reduction and calibration of the NIRSPEC data. In Section 4, we describe our NIR Doppler technique, the expected precision, and potential sources of noise that limit the overall precision we achieve. In Section 5, we discuss the overall statistical properties of our Doppler measurements. In Section 6, we describe four individual RV variables and present orbital solutions. In Section 7, we describe the rotational and kinematic properties of our sample and compare the distributions of these values to those in the literature. In Section 8, we estimate the rate of tight $(a<1 \mathrm{AU})$ UCD binaries and simulate the sensitivity of our survey to giant planet companions.

\section{SAMPLE SELECTION AND OBSERVATIONS}

Thanks to all-sky NIR surveys such as the Two Micron All Sky Survey (2MASS), Sloan Digital Sky Survey (SDSS), and DENIS, the L spectral class is a well-studied group of several hundred old low-mass stars and younger brown dwarfs. There is an inherent degeneracy between age and spectral type for these objects, but at field ages $(t>1 \mathrm{Gyr})$ the mid-L dwarfs are expected to be brown dwarfs, objects with masses below the minimum required for main-sequence hydrogen burning, while late-M and early-L dwarfs may be very small hydrogen burning stars (Burrows et al. 2001). The brown dwarfs slowly cool, radiating away their initial thermal energy over billions of years. With their cool temperatures, the atmospheres of UCDs contain a wide array of molecules, including $\mathrm{TiO}, \mathrm{VO}$, and $\mathrm{CO}$, as well as dust particles, leading to complex NIR spectra (Rayner et al. 2009).

We selected a sample of field UCD dwarfs brighter than $K=13.0$ observable from Mauna Kea (decl. $>-40^{\circ}$ ). Our targets and their observed properties are listed in Table 1. The majority of our targets are classified as L dwarfs, though three may be classified as early-L or late-M depending on the spectral diagnostics used. Today, our sample contains more than $70 \%$ of the known L dwarfs that satisfy our magnitude and declination limits (http://www.dwarfarchives.org), though a number of new $\mathrm{L}$ dwarfs were discovered during the course of our survey. Between 2003 March and 2009 May, we collected approximately 600 individual observations of a sample of 59 UCDs using the NIRSPEC (McLean et al. 1998) instrument on the Keck II telescope. NIRSPEC is a high-resolution, crossdispersed NIR echelle spectrograph and is a powerful instrument for high-resolution spectroscopy of cool stars and brown dwarfs. While NIRSPEC can be operated in conjunction with the adaptive optics (AO) system, our observations were obtained without $\mathrm{AO}$ since many of our targets are too faint for $\mathrm{AO}$ observations. We selected the 3 pixel (0'432) slit, the N7 order blocking filter, the thin IR blocker, and a spectrograph configuration designed to place our desired spectral region around the CO bandhead ( 2.285 to $2.318 \mu \mathrm{m}$ ) near the center of echelle order 33. This setup provided a resolution of $R=25,000$ and the 3 pixel slit was a good match for the typical seeing in $K$ band at Mauna Kea. Over the course of our survey we utilized this same spectrograph set-up and by using emission line lamps we were able to adjust the echelle and cross disperser angles in order to reproduce the positions of the echelle orders to within $\pm 0.07 \mathrm{~nm}$. NIRSPEC employs a $1024 \times 1024$ pixel ALADDIN InSb array with $27 \mu \mathrm{m}$ pixels and all of our science data were gathered using Fowler sampling (MCDS-16) readouts in order to reduce the read noise to the nominal level of $25 e^{-}$.

We gathered observations of our targets in nod pairs where the target was nodded along the slit by approximately $6^{\prime \prime}$ between the first and second exposures of a pair. This observing strategy facilitates the removal of sky emission lines through the subtraction of consecutive two-dimensional images. We selected integration times so as to achieve a signal-to-noise ratio $(\mathrm{S} / \mathrm{N})$ per pixel of between 50 and 100 in each of our individual extracted one-dimensional spectra with exposure times ranging from 500 to $1200 \mathrm{~s}$ per nod position. On each night, we gathered an extensive set of calibration data including a large number of 
Table 1

L Dwarf Sample

\begin{tabular}{|c|c|c|c|c|c|}
\hline ID & R.A. & Decl. & $J$ & $K$ & Sp. Type \\
\hline GJ1001B & 000434.84 & -404405.8 & 13.11 & 11.40 & L5 \\
\hline $2 \mathrm{M} 0015+35$ & 001544.76 & +351602.6 & 13.88 & 12.26 & L2 \\
\hline $2 \mathrm{M} 0036+18$ & 003616.17 & +182110.4 & 12.47 & 11.06 & L3.5 \\
\hline $2 \mathrm{M} 0045+16$ & 004521.43 & +163444.6 & 13.06 & 11.37 & L2 \\
\hline 2M0141+18 & 014103.21 & +180450.2 & 13.88 & 12.49 & L1 \\
\hline 2M0144-07 & 014435.36 & -071614.2 & 14.19 & 12.27 & L5 \\
\hline $2 \mathrm{M} 0213+44$ & 021328.80 & +444445.3 & 13.49 & 12.21 & L1.5 \\
\hline $2 \mathrm{M} 0227-16$ & 022710.36 & -162447.9 & 13.57 & 12.14 & L1 \\
\hline $2 \mathrm{M} 0228+25$ & 022811.01 & +253738.0 & 13.84 & 12.47 & L0 \\
\hline $2 \mathrm{M} 0235-23$ & 023559.93 & -233120.5 & 12.69 & 12.19 & L1 \\
\hline $2 \mathrm{M} 0251-03$ & 025114.90 & -035245.9 & 13.06 & 11.66 & L3 \\
\hline $2 \mathrm{M} 0306-36$ & 030611.59 & -364752.8 & 11.69 & 10.63 & M8 \\
\hline 2M0320-04 & 032028.39 & -044635.8 & 13.26 & 12.13 & M8/L0 \\
\hline $2 \mathrm{M} 0355+11$ & 035523.37 & +113343.7 & 14.05 & 11.53 & L5 \\
\hline $2 \mathrm{M} 0500+03$ & 050021.00 & +033050.1 & 13.67 & 12.06 & L4 \\
\hline $2 \mathrm{M} 0523-14$ & 052338.22 & -140302.2 & 13.08 & 11.64 & $\mathrm{~L} 2.5$ \\
\hline $2 \mathrm{M} 0539-00$ & 053952.00 & -005901.9 & 14.03 & 12.53 & L5 \\
\hline 2M0543+64 & 054318.87 & +642252.8 & 13.57 & 12.05 & L1 \\
\hline LSR0602+39 & 060230.45 & +391059.2 & 12.30 & 10.86 & L1 \\
\hline $2 \mathrm{M} 0632+83$ & 063206.17 & +830501.4 & 13.75 & 12.40 & L0.5 \\
\hline $2 \mathrm{M} 0652+47$ & 065230.73 & +471034.8 & 13.51 & 11.69 & L4.5 \\
\hline $2 \mathrm{M} 0700+31$ & 070036.64 & +315726.6 & 12.92 & 11.32 & L3.5 \\
\hline $2 \mathrm{M} 0717+57$ & 071716.26 & +570543.0 & 14.64 & 12.94 & L3 \\
\hline $2 \mathrm{M} 0746+20$ & 074642.56 & +200032.1 & 11.76 & 10.47 & L0.5 \\
\hline $2 \mathrm{M} 0828-13$ & 082834.19 & -130919.8 & 12.80 & 11.30 & L2 \\
\hline 2M0835-08 & 083542.56 & -08 1923.7 & 13.17 & 11.14 & L5 \\
\hline 2M0847-15 & 084728.72 & -153237.2 & 13.51 & 12.06 & L2 \\
\hline 2M0921-21 & 092114.10 & -210444.6 & 12.78 & 11.69 & L1.5 \\
\hline 2M0911+74 & 091112.97 & +740108.1 & 12.92 & 11.75 & L0 \\
\hline $2 \mathrm{M} 1022+58$ & 102248.21 & +582545.3 & 13.50 & 12.16 & L1 \\
\hline $2 \mathrm{M} 1045-01$ & 104524.00 & -014957.6 & 13.16 & 11.78 & L1 \\
\hline 2M1048+01 & 104842.81 & +011158.0 & 12.92 & 11.62 & L1 \\
\hline $2 \mathrm{M} 1108+68$ & 110830.81 & +683016.9 & 13.12 & 11.58 & L1 \\
\hline $2 \mathrm{M} 1112+35$ & 111225.67 & +3548 13.1 & 14.58 & 12.72 & L4.5 \\
\hline 2M1155-37 & 115539.52 & -372735.0 & 12.81 & 11.46 & $\mathrm{~L} 2$ \\
\hline $2 \mathrm{M} 1203+00$ & 120358.12 & +001550.0 & 14.01 & 12.48 & L3 \\
\hline $2 \mathrm{M} 1221+02$ & 122127.70 & +025719.8 & 13.17 & 11.95 & L0 \\
\hline 2M1300+19 & 130042.55 & +19 1235.4 & 12.72 & 11.62 & L1 \\
\hline $2 \mathrm{M} 1305-25$ & 130540.19 & -254105.9 & 13.41 & 11.75 & L2 \\
\hline $2 \mathrm{M} 1425-36$ & 142527.98 & -365022.9 & 13.75 & 11.81 & L3 \\
\hline 2M1439+19 & 143928.36 & +192914.9 & 12.76 & 11.55 & L1 \\
\hline $2 \mathrm{M} 1506+13$ & 150654.41 & +132106.0 & 13.36 & 11.74 & L3 \\
\hline $2 \mathrm{M} 1507-16$ & 150747.69 & -162738.6 & 12.83 & 11.31 & L5 \\
\hline $2 \mathrm{M} 1515+48$ & 151500.83 & +484741.6 & 14.11 & 12.50 & L6 \\
\hline 2M1539-05 & 153941.89 & -052042.8 & 13.92 & 12.57 & L4 \\
\hline $2 \mathrm{M} 1552+29$ & 155259.06 & +294848.5 & 13.48 & 12.02 & LO \\
\hline 2M1555-09 & 155515.73 & -095605.5 & 12.56 & 11.44 & L1 \\
\hline LSR1610-0040 & 161029.00 & -004053.0 & 12.91 & 12.02 & $\operatorname{sdM} 7$ \\
\hline $2 \mathrm{M} 1645-13$ & 164522.11 & -131951.6 & 12.45 & 11.15 & L1.5 \\
\hline $2 \mathrm{M} 1658+70$ & 165803.80 & +702701.5 & 13.29 & 11.91 & L1 \\
\hline 2M1705-05 & 170548.34 & -051646.2 & 13.31 & 12.03 & \\
\hline $2 \mathrm{M} 1731+27$ & 173129.74 & +272123.3 & 12.09 & 10.91 & L0 \\
\hline $2 \mathrm{M} 1807+50$ & 180715.93 & +501531.6 & 12.93 & 11.60 & L1.5 \\
\hline $2 \mathrm{M} 1821+14$ & 182128.15 & +141401.0 & 13.43 & 11.65 & L4.5 \\
\hline $2 \mathrm{M} 1854+84$ & 185445.97 & +842947.1 & 11.54 & 11.35 & $\ldots$ \\
\hline $2 \mathrm{M} 2036+10$ & 203603.16 & +105129.5 & 13.95 & 12.45 & L3 \\
\hline $2 \mathrm{M} 2057-02$ & 205754.09 & -025230.2 & 13.12 & 11.72 & L1.5 \\
\hline $2 \mathrm{M} 2104-10$ & 210414.91 & -103736.9 & 13.84 & 12.37 & L2.5 \\
\hline 2M2224-01 & 222443.81 & -015852.1 & 14.07 & 12.02 & L4.5 \\
\hline
\end{tabular}

Notes. Coordinates, magnitudes, and spectral types of the targets in our sample as gathered from http://www.dwarfarchives.com and cross checked against the database of UCDs recently published by Faherty et al. (2009). The spectral types listed here are those derived from optical diagnostics. Optical spectral types are not available for $2 \mathrm{M} 1705-05$ and $2 \mathrm{M} 1854+84$ but based on their broadband colors these objects are expected to be L dwarfs. flat field images and observations of bright, rapidly rotating A stars at a range of airmasses. The A star spectra are free from stellar absorption features in our spectral region and are therefore useful for monitoring changes in telluric absorption. During the course of our survey, we obtained between 2 and 16 epochs of observations for each target in our sample. This inhomogeneous pattern of visits was determined in part by the scheduling of our observing time, but objects were also prioritized based on their projected rotational velocity, $V \sin i$. Typically, we observed objects with large $V \sin i$, which limits our Doppler precision, only twice, while objects exhibiting clear evidence for Doppler variations were observed at every opportunity.

\section{DATA REDUCTION}

We reduced the NIRSPEC data and extracted spectra from order 33 using a set of custom IDL procedures developed for this survey to flat field the two-dimensional spectra, trace the spectral orders, and extract one-dimensional spectra. For each observing session, which we defined as the period between physical movements of the internal components of the spectrograph, sets of 20 flat field images were median combined to produce a "superflat." As a result of computer or hardware problems, there were occasionally multiple observing sessions defined within a single observing night. The individual flat fields have an integration time of $4 \mathrm{~s}$, resulting in an average signal of 4500 ADU per pixel in order 33. The ALADDIN detector has a small dark current $\left(0.2 e^{-1}\right.$ pixel $\left.^{-1} \mathrm{~s}^{-1}\right)$ so we also gathered $4 \mathrm{~s}$ dark frames for use in the creation of the superflats. Prior to median combination the individual flat fields were each normalized so as to compensate for changes in the overall flux levels due to warming of the flat-field lamp. We used the superflat to trace echelle order 33 and to define the position of the order across the detector. Since we replicated the same spectrograph configuration during each observing session, the positions of the echelle orders are known to within a few pixels a priori in all of our data.

We began the reduction and extraction procedures by subtracting nod pairs. Provided that changes in the detector or spectrograph properties were negligible over the timescale of the nod pair, this effectively removes the dark current and the bias level of the detector. If the sky brightness is not rapidly changing then night sky emission lines are also removed by pair subtraction. Following subtraction, the two resulting two-dimensional difference images $(\mathrm{A}-\mathrm{B}$ and $\mathrm{B}-\mathrm{A})$ were flat fielded using the superflat to compensate for sensitivity variations both across the order and at the pixel-to-pixel level, resulting in intensityrectified difference images. We trimmed 24 noisy columns from one edge of the detector, leaving a total of 1000 columns. In our description of the data, the echelle orders run roughly parallel to the rows of the detector. We extracted spectra from the intensityrectified difference images following the procedures outlined in Horne (1986). The position of the spectrum across order 33 was determined by fitting a Gaussian in the spatial direction at each column and then fitting the resulting centers to a fourth-order polynomial with outlier rejection. Using the difference image, we built an empirical model of the spectral profile within order 33 in the spatial direction. This model accommodates smooth variations in the width or shape of the profile across the order and is normalized so that the integral of the spectral profile at each column is unity. At each column, we fit this model spectral profile to the data by solving for the scale factor and an additive offset, to account for incomplete sky subtraction, that best fits 
the data in a least squares sense. The variance for each pixel was determined from the quoted gain and read noise estimated from a region of the NIRSPEC detector between the spectral orders. We found that the read noise estimated in this way was often close to $75 e^{-}$, much larger than the quoted value of $25 e^{-}$. The optimal estimate of the total flux at each of the 1000 columns was determined by the scale factor of the best-fit model spectral profile. Each profile fit was conducted iteratively to mitigate the effects of cosmic rays or bad pixels by rejecting outliers and then re-fitting.

Some of our NIRSPEC data exhibit a significant additional noise. A transient pattern is sometimes seen in a single quadrant of the NIRSPEC detector such that every eighth row has significantly enhanced noise. The phase of this pattern changes in time both within a night and between nights while the 8 pixel periodicity remains fixed. These noisy rows run roughly parallel to the echelle orders so, depending on the phase of the pattern, they can have a significant impact on the $\mathrm{S} / \mathrm{N}$ of the extracted data. The enhanced noise was seen in approximately $22 \%$ of our observations, though not always in the immediate vicinity of order 33. In a smaller subset of our data more complex noise patterns were seen with multiple patterns each having an 8 pixel period. We visually inspected all of the individual extracted spectra and culled approximately $10 \%$ as having poor $\mathrm{S} / \mathrm{Ns}$ or severe noise problems due to order 33 falling along a particularly noisy row.

\section{SPECTRAL MODELING}

We forward modeled the extracted spectra to measure the stellar RV and projected rotational velocity $(V \sin i)$. This procedure followed the methods described in Blake et al. (2007, 2008b) and is similar to that used by Butler et al. (1996) to obtain $3 \mathrm{~m} \mathrm{~s}^{-1}$ Doppler precision at optical wavelengths using an $I_{2}$ absorption cell. Our simultaneous calibrator is $\mathrm{CH}_{4}$ located not in a cell but in Earth's atmosphere. The basis for our modeling procedure is the interpolation and convolution of high-resolution spectral models to fit the lower resolution NIRSPEC data, which we denote $D$, by minimizing $\chi^{2}$. The basic form of our model can be expressed as

$$
M(\lambda)=\left(\left[L\left(\lambda \times\left(1+\frac{v}{c}\right)\right) \star K\right] \times T(\lambda)\right) \star \mathrm{LSF}
$$

where $\star$ indicates convolution, $L(\lambda)$ is a high-resolution UCD template, $K$ is the rotational broadening kernel, $T(\lambda)$ is the telluric spectrum, and LSF is the spectrograph line spread function. We began with a library of high-resolution synthetic template spectra computed as described in Marley et al. (2002) and Saumon \& Marley (2008). The models apply the condensation cloud model of Ackerman\& Marley (2001) with a sedimentation parameter of $f_{\text {sed }}=3$, corresponding to a moderate amount of condensate settling. The models used here have solar metallicity (Lodders 2003), use the opacities described in Freedman et al. (2008), have a fixed gravity of $\log g=5$ (cgs), and cover a range of $T_{\text {eff }}$ from 1200 to $2400 \mathrm{~K}$. The synthetic spectra provide monochromatic fluxes spaced $4.2 \times 10^{-6} \mu \mathrm{m}$ apart. We incorporated line broadening due to stellar rotation by convolving with the kernel defined by Gray (1992) using a linear limb-darkening parameter of 0.6 as appropriate for cool stars at infrared wavelengths (Claret 2000). We also used a highresolution $\left(5 \times 10^{-6} \mu \mathrm{m}\right.$ spacing $)$ telluric spectrum derived from observations of the Sun provided by Livingston \& Wallace

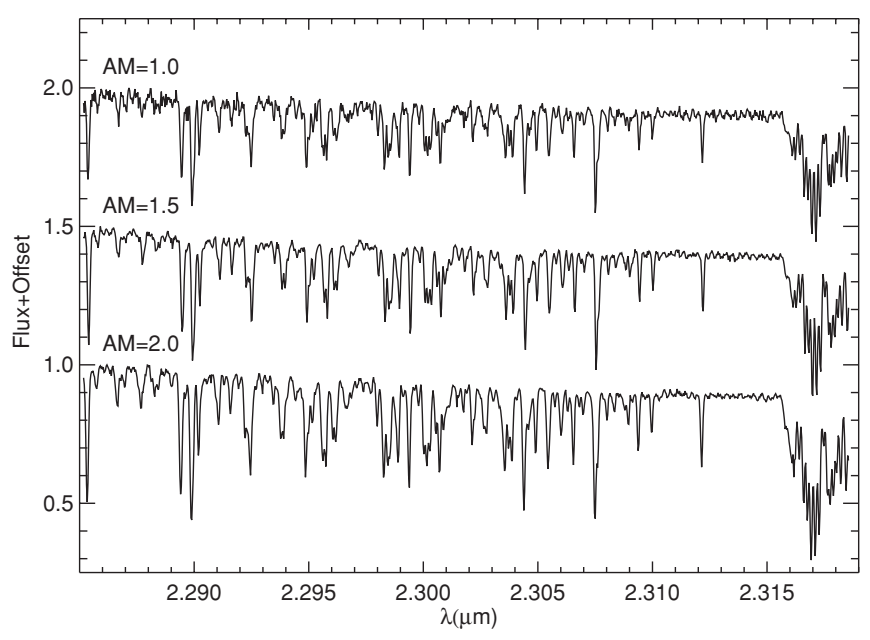

Figure 1. Examples of NIRSPEC A star spectra at three different airmasses. These absorption features, due mainly to $\mathrm{CH}_{4}$, serve as a simultaneous wavelength reference. The depths of the lines increase as the optical depth of the atmosphere increases with higher airmasses. The ringing between 2.310 and $2.315 \mu \mathrm{m}$ is the fringing pattern discussed in Section 5.1.

(1991). Using quadratic interpolation, we placed the synthetic UCD template and telluric model onto an evenly spaced wavelength grid with $5 \times 10^{-6} \mu \mathrm{m}$ spacing, about five times finer than the NIRSPEC data. We convolved the product of the rotationally broadened UCD template and the telluric model with an estimate of the spectrograph LSF. Finally, we used quadratic interpolation to place the model on the lower resolution NIRSPEC wavelength grid, which we define through polynomial mapping of pixel to wavelength. In total, the model of each individual spectrum has the following eleven free parameters: four for the polynomial mapping of pixel to wavelength, one for an overall flux scaling, four for a flux gradient across the spectrum, one for the LSF FWHM under the assumption that the LSF is a normalized Gaussian, and one for the UCD RV. We also had fixed parameters for the scaling of the telluric model with airmass, which we define later in this section, as well as the $V \sin i$ and effective temperature, $T_{\text {eff }}$, for each UCD, which we determined separately and fixed in all subsequent analyses. We fit our model to the NIRSPEC data in a least squares sense using an implementation of the AMOEBA (Nelder \& Mead 1965; Press et al. 1986) fitting method to minimize $\chi^{2}$.

We began our analysis of the NIRSPEC data by selecting a training sample of 200 A star observations acquired over the course of the survey at a range of airmasses from 1.0 to 2.0. Examples of A star spectra at a range of airmasses are shown in Figure 1. We modeled these observations without including the UCD template in order to refine our fitting procedure, determine the overall distribution of the best-fit wavelength and LSF parameters, and model the changes of the depths of telluric lines with airmass. Starting from the nominal 10 model parameters (no RV), we added an additional free parameter for the scaling of the depths of the telluric lines with airmass. The Livingston \& Wallace (1991) data are at airmass (AM) of 1.5, but our data were acquired at a wider range of airmasses. At higher airmass the optical depth of the atmosphere increases and we expect the telluric line depths to increase. We assumed a one-parameter scaling of the depths of the telluric lines from the AM $=1.5$ model $\left(T_{0}\right)$ with airmass $T=T_{0}^{\tau}$. Based on the fits to the A star training sample, we found that the telluric line depths observed at the summit of Mauna Kea, shown in Figure 2, were well fit with $\tau=\mathrm{AM} \times 0.432$. After this initial determination of 


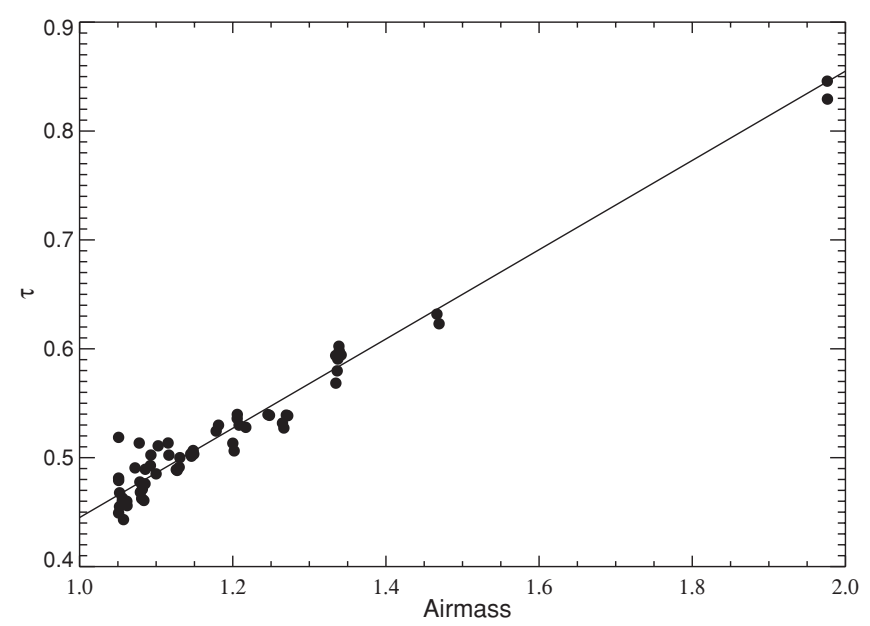

Figure 2. Results of fits to A star spectra for the single parameter, $\tau$, that is used to scale the depths of the lines in the telluric template with airmass.

the telluric scaling, we adopted this relation for $\tau$ for all of the subsequent fitting of NIRSPEC spectra and fixed this parameter in the modeling of each UCD spectrum.

In order for our fitting procedure to successfully converge on the correct model parameters, excellent initial estimates of the parameters were required. The A star training sample allowed us to determine the average model parameters related to the spectrograph and select good initial values for each parameter. We found that our nominal model resulted in A star fits with $\chi_{v}^{2} \sim 2-4$. Adding additional parameters for the wavelength solution did not significantly improve the overall quality of the fits. We also found that conducting an initial cross-correlation of the first 200 pixel chunk with a nominal model of an NIRSPEC A star observation allowed for the determination of the zerothorder term in the wavelength solution to better than 0.1 of an NIRSPEC pixel, sufficiently precise for AMOEBA to reliably converge on the correct wavelength solution. We investigated how the LSF changes across the spectral order by fitting portions of A star spectra independently. While the FWHM of the
Gaussian LSFs across the spectra change with time, we found that the ratios of the FWHM of the best-fit Gaussian LSFs in different portions of the spectra were relatively constant so that a single fixed parameter could be used to describe the slow change in the width of the LSF across the order. By looking at the average residuals of the fits to all 200 A stars, we also identified individual telluric lines that did not follow the scaling with airmass. In Figure 3, we show four lines that are likely not $\mathrm{CH}_{4}$ absorption features but may be features due to $\mathrm{H}_{2} \mathrm{O}$ (Rothman et al. 2009). We excluded a small spectral region (10 pixels) around each of these features in all of our fitting of the UCD spectra by assigning zero statistical weight.

The residuals of the model fits to the A star sample are shown in Figure 3. With our instrumental setup a fringe-like modulation was often seen in the extracted spectra and is clearly visible in the A star residual around $2.314 \mu \mathrm{m}$ in Figure 1. This pattern is likely due to an internal reflection in an optical element near the detector (Brown et al. 2003) and is generally described as the superposition of sinusoidal patterns with amplitudes of roughly $1 \%$ and periods of approximately $0.3 \mathrm{~nm}$. An additional complication is that the spatial frequency of the fringe pattern is similar to the spatial frequencies of absorption features seen in slowly rotating UCDs, so it cannot be easily removed without degrading the signal that we wish to model. Like Brown et al. (2003), we found that the fringing pattern varies somewhat over time in phase, frequency, and amplitude. Assuming that the fringe signal is multiplicative (as opposed to additive), we built a model of the average fringe signal in wavelength space by averaging the A star residuals (Data/Model) in bins of width $0.03 \mathrm{~nm}$. This model, shown in Figure 3, is fixed in wavelength space and has an amplitude of approximately $1 \%$ and is quasi-periodic with a dominant period of $0.3 \mathrm{~nm}$. Including this fringe model in the fitting of the A star sample resulted in significant improvements in $\chi^{2}$ for the highest $\mathrm{S} / \mathrm{N}$ spectra though had negligible impact on the resulting overall distribution of the best-fit model parameters. Given that the fringe pattern is a quasi-periodic multiplicative modulation it is possible that the average shapes of the telluric lines, and

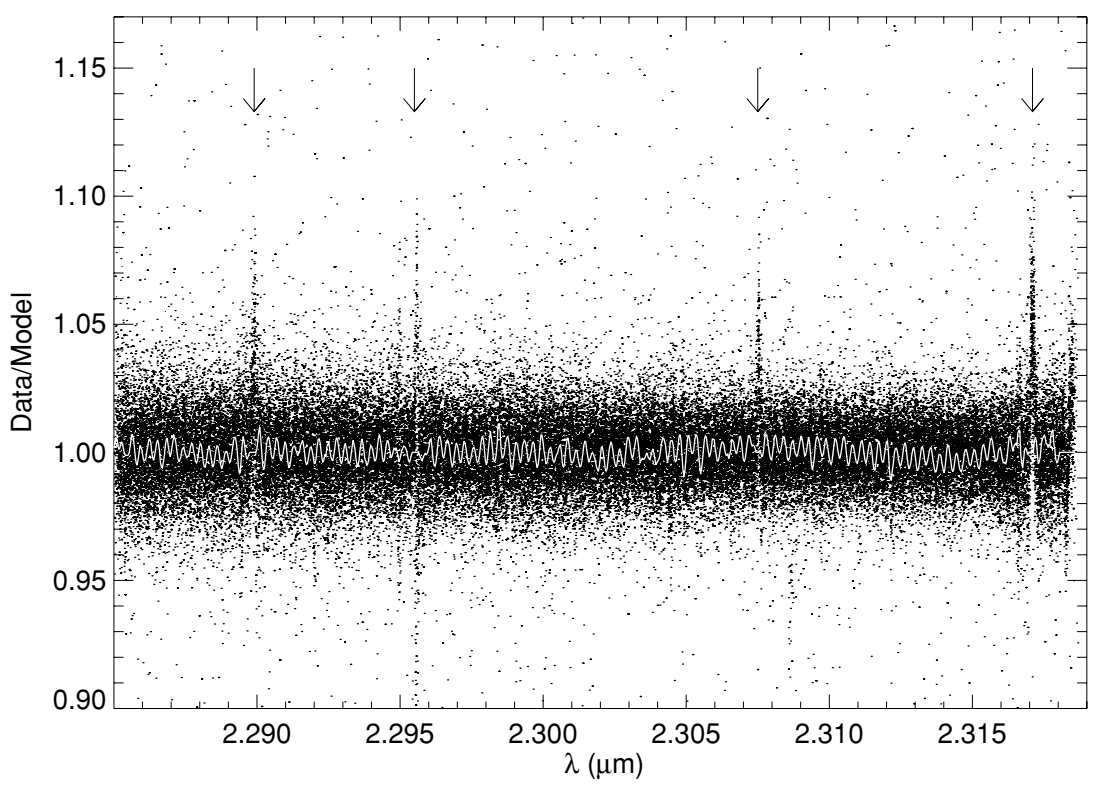

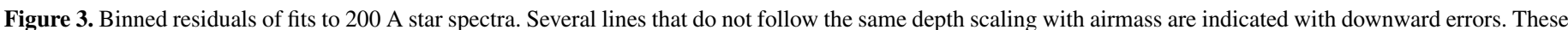

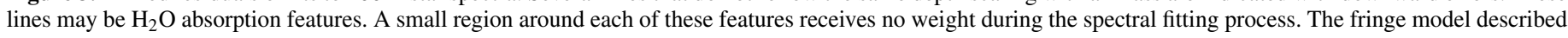
in Section 4 is overplotted in white. 


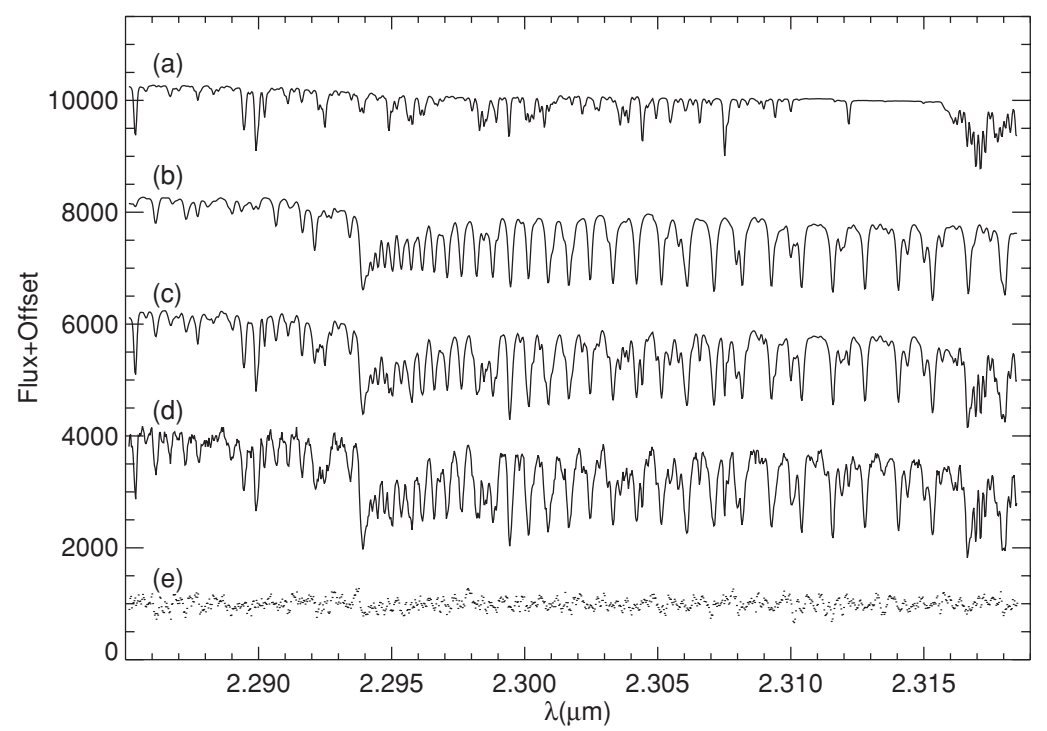

Figure 4. Schematic example of the forward modeling process described in Section 4: (a) observed telluric spectrum, (b) synthetic L dwarf spectrum broadened to account for stellar rotation, (c) model from product of (a) and (b) convolved with the spectrograph LSF, (d) NIRSPEC observation of 2M1048+01, (e) residuals of model fit to data.

therefore the resulting wavelength solutions, could be biased as a function of the relative phase of the fringe and the telluric absorption features. At the same time, the fact that the fringe model is not strictly periodic could mean that this effect averages out over the spectrum, reducing the impact on the resulting fits. Given the small amplitude of the fringe model compared to the $\mathrm{S} / \mathrm{N}$ of our A star observations $(\mathrm{S} / \mathrm{N} \sim 200)$ it is perhaps not surprising that it is not an important factor in our fits.

We fit the UCD spectra following an iterative process using the average parameter values determined from the A star analysis as the starting parameters for AMOEBA. As with the A star analysis, we used the first 200 pixel chunk of each spectrum to estimate the zeroth-order term of the wavelength solutions by cross-correlating against a nominal telluric model. This first 200 pixel chunk $(2.585-2.592 \mu \mathrm{m})$ of the UCD spectrum is relatively devoid of stellar absorption features and so fitting this chunk to the telluric-only model was useful both for determining the starting wavelength position as well as the single LSF FWHM parameter. We also produced an initial RV estimate by cross-correlating a spectral region with rich CO features $(2.298-2.305 \mu \mathrm{m})$ against a fiducial UCD model at zero velocity. This step, which we found necessary for ensuring convergence of the fitting process, provides a rough $\left( \pm 10 \mathrm{~km} \mathrm{~s}^{-1}\right)$ estimate of the RV that includes shifts due to barycentric motion. We estimated the $T_{\text {eff }}$ and $V \sin i$ for each UCD in a two-step process. We began by fitting each spectrum of each object to a grid of 360 UCD models spanning $1200 \mathrm{~K}<T_{\text {eff }}<2400 \mathrm{~K}$ and $9 \mathrm{~km} \mathrm{~s}^{-1}<V \sin i<100 \mathrm{~km} \mathrm{~s}^{-1}$. For each NIRSPEC spectrum, we found the global minimum $\chi^{2}$ in the $T_{\text {eff }}$ and $V \sin i$ grid resulting in one estimate of each parameter for each spectrum. Using these initial estimates we set a single $T_{\text {eff }}$ for each object by selecting the UCD model that produced the lowest average $\chi^{2}$ values over all of the spectra of the object. With $T_{\text {eff }}$ fixed for each object, we ran a second set of fits over a finer grid in $V \sin i$ and then fit for the minimum of the resulting curve of $\chi^{2}$ as a function of $V \sin i$ to estimate the best-fit $V \sin i$ for each spectrum. We adopted the simple average of the individual estimates from each spectrum as the fixed $V \sin i$ of the UCD and the scatter of those estimates as the error on the $V \sin i$. These two parameters, $T_{\text {eff }}$ and $V \sin i$, were then fixed for all subsequent analyses. We determined the lower limit on $V \sin i$, set by the resolution of NIRSPEC, by estimating the value below which changes in $V \sin i$ did not improve the $\chi^{2}$ of the fits to UCDs that are known to be slow rotators.

With the global UCD parameters fixed ( $V \sin i$ and $T_{\text {eff }}$ ) for each target, we estimated the RV of each observation of each target using the same fitting process. Each spectrum was fit in two stages, rejecting outliers following an initial fit and then fitting again using the best-fit parameters from the first fit as the new starting parameters. We conducted extensive tests using artificial spectra to ensure that the correct minima were being found by AMOEBA by conducting fits with fixed wavelength solutions over a large, high-resolution parameter space. We generated the artificial spectra based on the wavelength solutions found in the A star analysis, the synthetic UCD templates over a range of $T_{\text {eff }}$ and $V \sin i$, and noise properties representative of the actual NIRSPEC data. We found that with good starting values our AMOEBA fitting procedure reliably converged on the true minimum of $\chi^{2}$ and the correct model parameters. The formal reduced $\chi^{2}$ of the UCD fits fall in a wide range $\left(2<\chi_{v}^{2}<8\right)$. Unlike with the A star analysis, where the $\chi^{2}$ is likely dominated by the noise properties of the NIRSPEC detector and limitations in our LSF model, the UCD fits may be dominated by the mismatch between the theoretical stellar templates and the UCD and we expect some larger values of $\chi^{2}$. For example, systematic discrepancies are sometimes seen in the structure of the $\mathrm{CO}$ bandhead or in the depths of individual $\mathrm{CO}$ features longward of the bandhead. We emphasize that systematic deviations between the spectra and the theoretical models may not be shortcomings of the models themselves but rather a symptom of the small range of our library of synthetic templates in terms of $\log g, f_{\text {sed }}$, and metallicity. An example of an NIRSPEC spectrum, the best-fit model, and the residuals is shown in Figure 4 and the top panel of Figure 9.

We estimated the statistical uncertainty on each RV measurement, $\sigma_{\mathrm{RV}}$, based on the photon-limited Doppler precision (PLDP) presented in Butler et al. (1996)

$$
\sigma_{R V}=\left[\Sigma\left(\frac{d M / d \mathrm{RV}}{\epsilon_{D}}\right)^{2}\right]^{-1 / 2}
$$




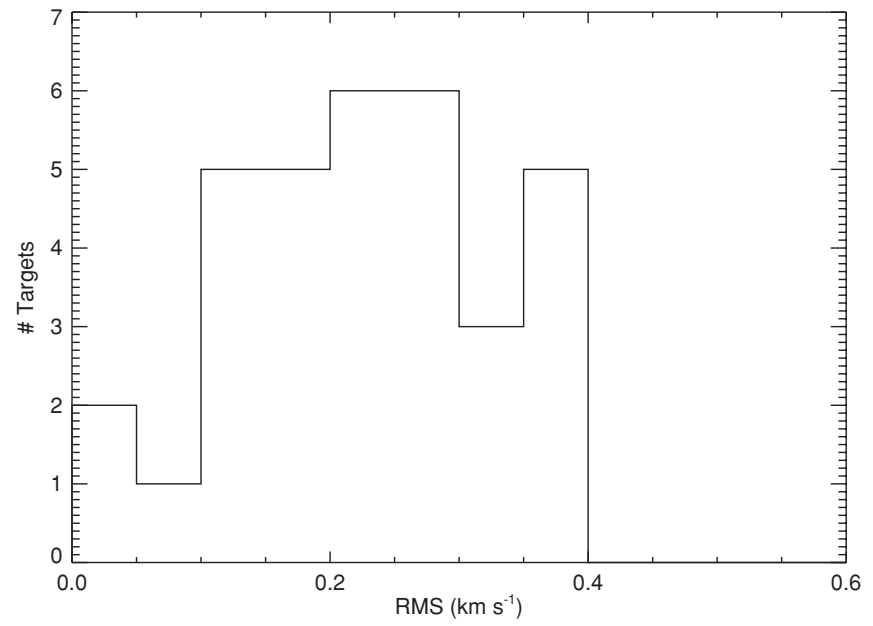

Figure 5. Histogram of the measured RV rms dispersion of the UCDs with observations on three or more epochs and $V \sin i<30 \mathrm{~km} \mathrm{~s}^{-1}$. For the majority of our targets, we achieve an overall precision of $100-300 \mathrm{~m} \mathrm{~s}^{-1}$.

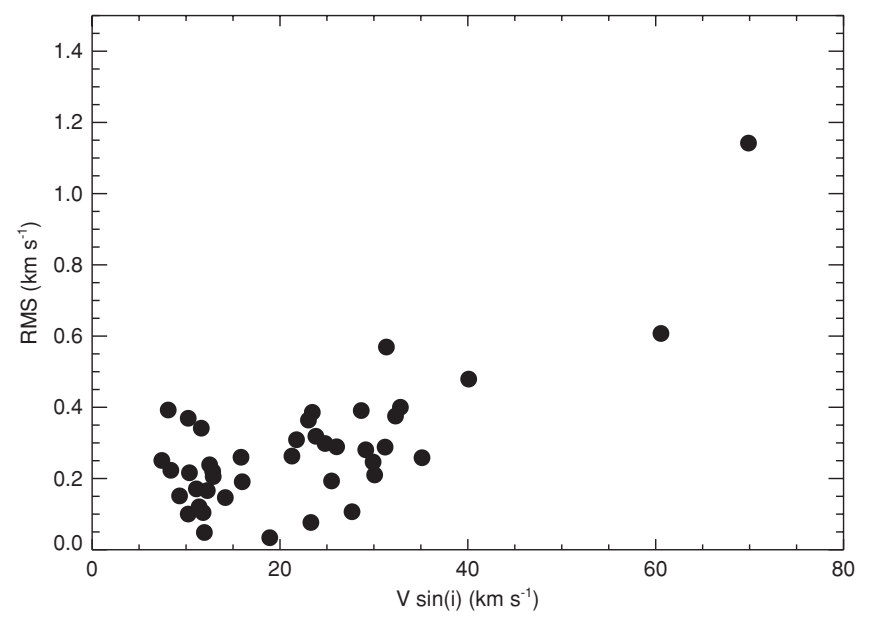

Figure 6. Estimated RV rms dispersions for targets with observations on three or more epochs. Rapid rotation broadens the spectral features of the $\mathrm{L}$ dwarfs, decreasing the expected RV precision. We see rms below $200 \mathrm{~m} \mathrm{~s}^{-1}$ in a number of our slowly rotating targets. We estimate that the minimum $V \sin i$ measurable with our NIRSPEC data is $9 \mathrm{~km} \mathrm{~s}^{-1}$.

where the sum is over all pixels, $d M / d \mathrm{RV}$ is the rate of change of the model flux at a given pixel in velocity units, and $\epsilon_{D}$ is a fractional noise term. Here, $M$ is the UCD (or telluric) component of the best-fit model for each spectrum. In the case of photon noise alone $\epsilon_{D}=\sqrt{D \times G} /(D \times G)$, where $D$ is the data in $\mathrm{ADU}$ and $G$ is the detector gain. We estimated the PLDP of the best-fit model by evaluating the telluric and UCD components of the model separately and adding the two error estimates in quadrature. This accommodates the case of a high-S/N observation of a rapidly rotating object where the wavelength solution may be determined precisely from the deep telluric lines but the RV is only poorly determined from the broad stellar features. For the typical S/N of our UCD observations, we estimate that the telluric lines themselves limit the RV precision to $\sigma_{\mathrm{RV}}>60 \mathrm{~m} \mathrm{~s}^{-1}$. Our data have significant sources of noise beyond just photon noise. Instead of assuming photon noise alone, we estimated the noise term $\epsilon_{D}$ from the residuals of the best-fit model $[S=D-M]$. The residuals could be dominated by systematic discrepancies between the model and the data, which could result in an overestimation of the noise term based on the rms of $S$ alone. To remove such systematic residuals, we

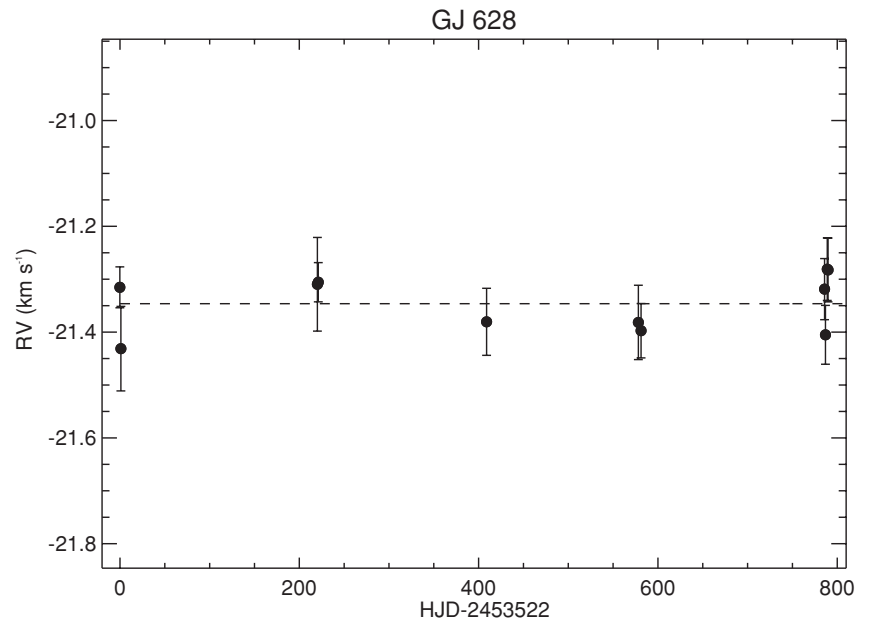

Figure 7. RV measurements of the bright, slowly rotating M dwarf GJ 628. We estimate the rms to be $50 \mathrm{~m} \mathrm{~s}^{-1}$ over the 800 day span of the observations. The scatter in these measurements is fully consistent with the photon-limited Doppler precision error estimate given by Equation (2).

first smoothed the residuals $\left[S^{\prime}=\operatorname{SMOOTH}(S)\right]$ with a boxcar filter of width 5 pixels and then estimated the noise term

$$
\epsilon_{D}=\frac{\sqrt{\left\langle\left(S-S^{\prime}\right)^{2}\right\rangle-\left\langle S-S^{\prime}\right\rangle^{2}}}{D}
$$

We applied barycentric corrections to the individual RV estimates calculated using the code bcvcorr (G. Torres 2007, private communication) and then combined observations (generally nod positions A and B) from the same epoch using a weighted mean.

\section{RADIAL VELOCITY PRECISION}

Based on the standard deviations of the RV measurements of 43 of our targets with observations on three or more epochs, $V \sin i<30 \mathrm{~km} \mathrm{~s}^{-1}$, and excluding known or suspected variables, shown in Figure 5, we estimate our RV precision to be approximately $100-300 \mathrm{~m} \mathrm{~s}^{-1}$ for slowly rotating UCDs. In Figure 6, we compare the measured standard deviation of the RVs of each of our targets to the estimated $V \sin i$. As expected, our RV precision degrades significantly for rapidly rotating $\mathrm{L}$ dwarfs. During the course of our survey, we also observed the bright $(K=5.08)$, slowly rotating $\left(V \sin i<8 \mathrm{~km} \mathrm{~s}^{-1}\right)$ M3 dwarf GJ 628 as an RV standard. This object is more massive than the stars in our UCD sample, falling outside the $T_{\text {eff }}$ range of our synthetic templates. For the spectral fitting, we used a synthetically generated $\mathrm{M}$ dwarf template with $T_{\text {eff }}=3400 \mathrm{~K}$, $\log g=4.8$ computed from updated and improved NextGen (Hauschildt et al. 1999) models (T. Barman 2008, private communication) From our analysis of 11 epochs of observations of GJ 628, we found an RV rms of $50 \mathrm{~m} \mathrm{~s}^{-1}$ over a period of 800 days, as shown in Figure 7 . We note that the scatter of these RV measurements is well described by the PLDP error estimates obtained using the technique described in Section 4 $\left(P\left(\chi_{\mathrm{RV}}^{2} \leqslant\right)=0.47\right)$ indicating that at least for bright, slowly rotating objects we are achieving an RV precision very close to the photon limit.

To reliably estimate the statistical significance of any RV variations we detect in our UCD sample it is necessary to understand the underlying errors, both systematic and statistical, on our individual measurements. We have estimated the statistical errors on our individual RV measurements, $\sigma_{\mathrm{RV}}$, directly from the data, 


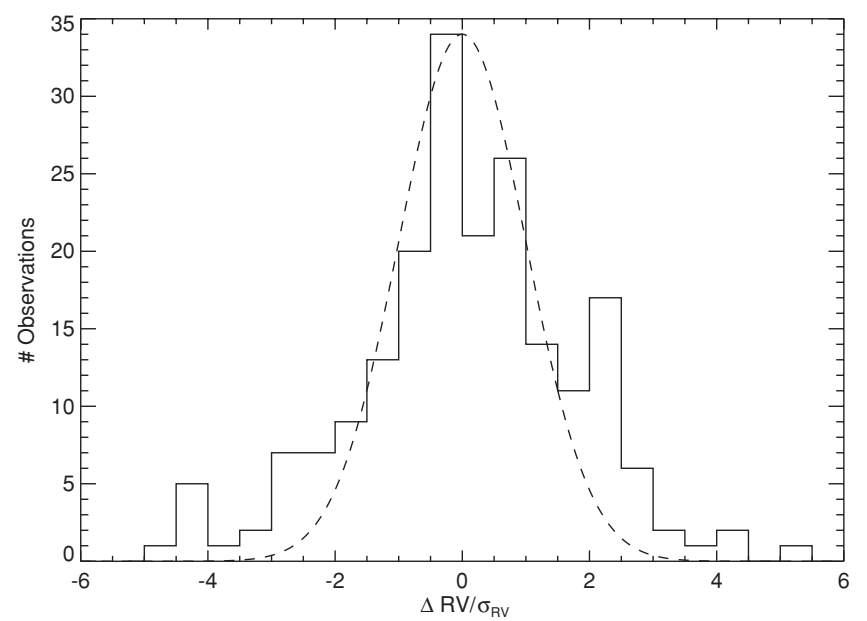

Figure 8. Overall distribution of $\Delta \mathrm{RV}$ normalized by the PLDP error $\sigma_{\mathrm{RV}}$. Here, we have assumed that all of the objects in this sub-sample have no intrinsic RV variations. The dashed line is an $N[0,1]$ distribution, the expected distribution if the error estimates correctly describe the data. We scale the empirical error estimates, $\sigma_{\mathrm{RV}}$, by a factor of 1.9 in order to make them consistent with the expected $N[0,1]$ distribution.

but these estimates do not take into account systematic effects that may occur between epochs. To investigate the overall statistical properties of our measurements we selected a sub-sample of objects that have observations on three or more epochs and are not known or suspected binaries. Assuming the null hypothesis that each of these UCDs has a constant RV, using the empirically determined internal errors, $\sigma_{\mathrm{RV}}$, for each of the 207 measured $\mathrm{RVs}$, we calculated $\chi_{\mathrm{RV}}^{2}=591$ for $207-43=164$ degrees of freedom (assuming one parameter for the constant RV of each target). If we have excluded all of the actual RV variables from this analysis, then this value of $\chi_{\mathrm{RV}}^{2}$ indicates that our statistical error estimates are too small and that there is a significant systematic contribution to be included in our overall error model. The overall error distribution for this sub-sample is shown in Figure 8, which shows that there are significant non-Gaussian tails at $|\Delta \mathrm{RV}|>2 \sigma$. We scaled all of the PLDP error estimates for the UCDs by a factor of $\sqrt{\chi_{v}^{2}}=1.9$, resulting in a reduction of the $\chi_{\mathrm{RV}}^{2}$ to 163.6 for the same number of degrees of freedom $\left[P\left(\chi_{\mathrm{RV}}^{2} \leqslant 163.6\right)=0.50\right]$. In some cases it is clear that the scaled errors are too large. For example, the scatter about the best-fit orbital solution for the binary 2M0320-04, described in Section 6.1, is $135 \mathrm{~m} \mathrm{~s}^{-1}$ while the smallest error estimate for a single point in the fit is $178 \mathrm{~m} \mathrm{~s}^{-1}$. Despite this possible overestimation, we used the scaled RV error estimates for all subsequent analyses of the UCD RV measurements. Based on our observations of the RV standard GJ 628, we conclude that our modeling process can produce RV measurements near the photon limit over long timescales and that the worse overall RV precision obtained for the UCDs could be due to a number of factors. The tails of the distribution of $\Delta R V$ could be the result of real RV variability since, we have only excluded known and suspected binaries from our sub-sample. While the theoretical template used to model GJ 628 is a very good match for its spectrum, mismatch between the theoretical templates and the actual spectra of the UCDs could lead to worse RV precision. Lastly, it is possible that the much longer integration times used for the UCD observations compared to the GJ 628 observations lead to systematic effects that are not encompassed in our model.

\subsection{Additional Tests}

In an effort to increase the overall precision of our RV measurements, as well as to address some technical issues that may be important for efforts to achieve high RV precision in the NIR with NIRSPEC or similar instruments, we experimented with some modifications to the standard fitting process described in Section 4. The first was the inclusion of the fringe model derived from observations of A stars into the UCD fits. Including this fringing model in the A star fitting process led to significant improvements to the resulting $\chi^{2}$, though it had negligible impact on the overall statistical properties of the resulting model parameters. Similarly, including the fringe model in the analysis of the GJ 628 observations did not result in a statistically significant decrease in the resulting RV scatter. While the A star and GJ 628 observations typically have $\mathrm{S} / \mathrm{N} \sim 200$, the UCD observations have $50<\mathrm{S} / \mathrm{N}<100$. The low-amplitude flux modulation of the fringe pattern is small compared to the read noise and photon noise and so was not expected to significantly impact the fitting process. We did experiment with including the fringe model in the flitting of the UCD spectra and found that the overall statistical properties of the resulting RV measurements were consistent with or without the fringe model and that the photon-limited errors still needed to be scaled by a factor of 1.9 to account for the observed scatter in the RV measurements of individual objects. The fringe model was not included in the final UCD RV results presented here.

While our theoretical templates are generally a very good match for the UCD spectra, for some individual objects there are significant systematic discrepancies in the shapes of the spectral features. This is most likely due to the fact we are only fitting a small library of synthetic templates to our spectra, and a wider range of $\log g, f_{\text {sed }}$, metallicity values, and line damping treatments could significantly improve the fits for some objects. If we have multiple observations of an object, and we can assume that the object has a constant RV, then it is possible to build an empirical spectral template from the observations themselves. We began by fitting the spectra using the theoretical templates following the standard procedure described in Section 4. Assuming that the wavelength solutions were sufficiently well determined by this initial fit, we divide the data by the telluric component of our best-fit model, resulting in a normalized UCD spectrum that is free from atmospheric absorption features. We corrected the wavelength solutions for the known barycentric velocity of each spectrum and then averaged all of the normalized spectra in $0.01 \mathrm{~nm}$ bins to create an empirical spectral template. For a slowly rotating UCD, where $V \sin i$ is comparable to or smaller than the spectrograph resolution, it is necessary to account for the broadening of the stellar spectral features by the LSF of the spectrograph. To do this, we assumed a Gaussian LSF with FWHM $=0.076 \mathrm{~nm}$ and carried out an iterative deconvolution following Lucy (1974) on the empirical template in an attempt to recover the unbroadened spectrum. Using this method, we created an empirical template for the rapidly rotating $\left(V \sin i=30.1 \mathrm{~km} \mathrm{~s}^{-1}\right) \mathrm{L}$ dwarf $2 \mathrm{M} 0652+47$ and found that the overall fit residuals were reduced to the level of $1 \%$. We also generated templates for more slowly rotating objects and a comparison between a theoretical template and an empirical template for the slowly rotating $\mathrm{L}$ dwarf 2M0835-08 is shown in Figure 9. While this technique did in some cases result in templates that were significantly better fits to the observed spectra, there are a number of drawbacks. The first is that a large number of spectra are required to produce the empirical template, preferably more than five, 


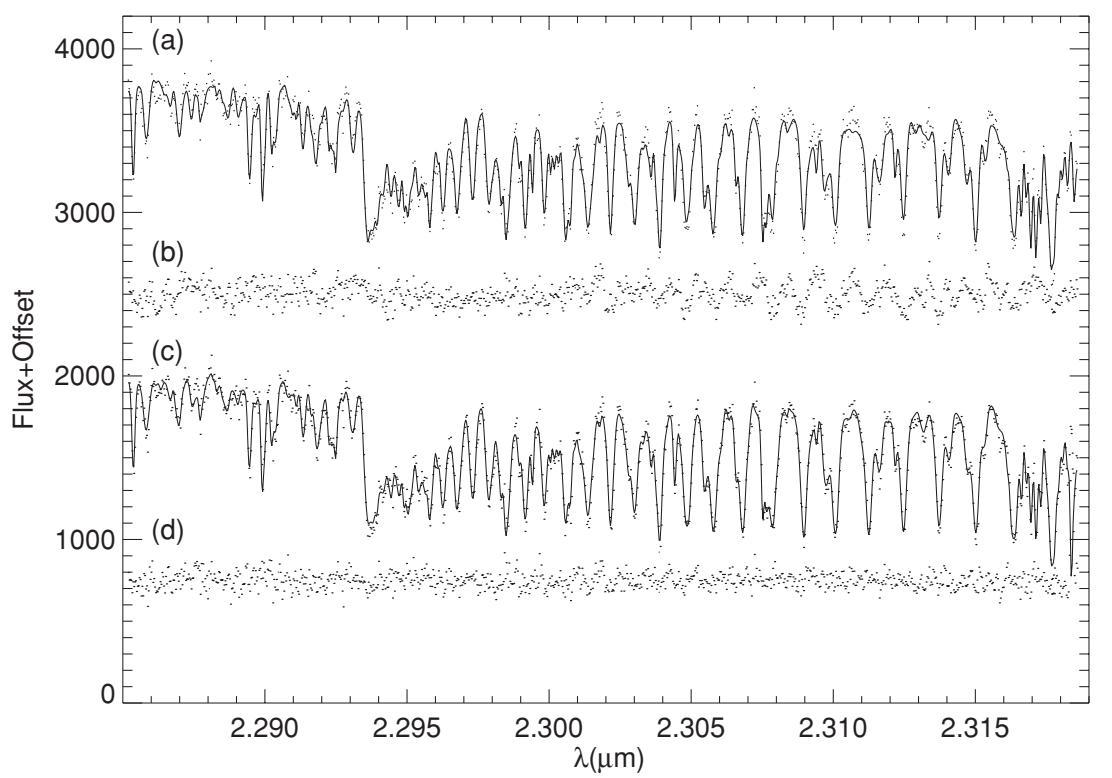

Figure 9. Comparison between fits for 2M0835-08 with theoretical and empirical templates: (a) NIRSPEC observation (points) with the best-fit model based on theoretical template (solid line). (b) Residuals of this fit. (c) NIRSPEC observation (points) with the best-fit model based on empirical template described in Section 5.1 (solid line); (d) residuals of this fit. The empirical template significantly improves the overall quality of the fit $\left(\Delta \chi^{2}=420\right)$ but in our tests the empirical templates did not result in an overall improvement in RV precision.

in order to robustly average in wavelength bins. The second is that we began by assuming that the object has a constant RV. Intrinsic Doppler shifts will result in a broadened spectral template and, particularly if the number of spectra is small, the empirical template may result in biased RV estimates. We found that using empirical templates to fit the subset of our sample with observations on five or more epochs did not yield RVs with a smaller dispersion. With our current data the creation of empirical templates may be most useful when looking for temporal changes in the residuals of the fits, which could be evidence for a faint companion.

As the precision of RV measurements at optical wavelengths has improved to the level of $1 \mathrm{~m} \mathrm{~s}^{-1}$ it has become clear that modeling the shape of the spectrograph's LSF is critically important. Butler et al. (1996) used a basis set of 11 Gaussian functions to model the subtle changes in the LSF asymmetries in their $I_{2}$ cell observations. Spectrograph LSF asymmetries may be inherent to the instrument or, in a slit spectrograph, they may arise from guiding errors inducing variations in the stellar position along the slit. We modeled the NIRSPEC LSF as a symmetric, Gaussian function that has a linear variation in width across the order. We experimented extensively with using multiple Gaussians to describe a more complex LSF in a manner similar to Butler et al. (1996). Owing to the limited S/N of our data, we could not reliably model the LSF asymmetry in either the A star or UCD spectra. Future improvements in the RV precision obtained using this technique will require a detailed modeling of the spectrograph LSF but the current spectral resolution and $\mathrm{S} / \mathrm{N}$ may not motivate such an analysis.

\section{RADIAL VELOCITY VARIABLES}

The measurements from our NIRSPEC survey, listed in Table 2, represent the largest available sample of highresolution, high-S/N, NIR observations of L dwarfs. The primary goal of this survey has been the detection of RV variations due to unseen companions. With an overall RV precision of approximately $200 \mathrm{~m} \mathrm{~s}^{-1}$ for slowly rotating UCDs, we are
Table 2

Table of RV Measurements

\begin{tabular}{lccc}
\hline \hline Object & HJD-2400000 & $\begin{array}{c}\mathrm{RV} \\
\left(\mathrm{km} \mathrm{s}^{-1}\right)\end{array}$ & $\begin{array}{c}\sigma \\
\left(\mathrm{km} \mathrm{s}^{-1}\right)\end{array}$ \\
\hline GJ1001B & 53271.94 & 32.95 & 0.70 \\
GJ1001B & 53272.88 & 33.28 & 0.38 \\
GJ1001B & 53328.74 & 32.83 & 0.43 \\
GJ1001B & 53669.82 & 32.60 & 0.27 \\
$\ldots$ & & & \\
\hline
\end{tabular}

(This table is available in its entirety in a machine-readable form in the online journal. A portion is shown here for guidance regarding its form and content.)

sensitive to many types of binary systems. The RV semiamplitude, $K_{1}$, of the primary (more luminous) star in a spectroscopic binary (or planetary system) is

$$
\begin{aligned}
K_{1} \approx & 654 \mathrm{~m} \mathrm{~s}^{-1}\left(\frac{P}{3 \mathrm{~d}}\right)^{-1 / 3}\left(\frac{M_{2}}{M_{\mathrm{J}}}\right)\left(\frac{M_{1}+M_{2}}{0.1 M_{\odot}}\right)^{-2 / 3} \\
& \times\left(1-e^{2}\right)^{-1 / 2} \sin i .
\end{aligned}
$$

The targets in our sample are expected to have masses from near $0.1 M_{\odot}$ down to the late- $\mathrm{L}$ dwarfs at $0.05 M_{\odot}$ (Burrows et al. 2001). If our survey is sensitive to signals with $\Delta R V>1 \mathrm{~km} \mathrm{~s}^{-1}$, then we may detect systems ranging from equal-mass binaries with periods of up to a decade to giant planetary companions with orbital periods of several weeks or less. We expect that the majority of our targets are not spectroscopic binaries (Allen 2007) and therefore will not exhibit RV variations. With the scaling of our PLDP error estimates our RV measurements are fully compatible with this hypothesis. The calculated $\chi_{\mathrm{RV}}^{2}$ for each UCD, assuming constant RV, and the corresponding probabilities are given in Table 3. We identified five with large $\chi_{\mathrm{RV}}^{2}$ having probabilities $P\left(\chi_{\mathrm{RV}}^{2} \leqslant\right)$, the statistical probability of getting a smaller value of $\chi_{\mathrm{RV}}^{2}$, greater than 0.999 . We note that three of these systems exhibiting significantly large $\chi_{\mathrm{RV}}^{2}$ are either known or candidate binaries. Two of these 
Table 3

Measured Properties of L Dwarfs

\begin{tabular}{|c|c|c|c|c|c|c|c|}
\hline ID & N Obs. & $\begin{array}{c}\Delta T \\
\text { (days) }\end{array}$ & $\begin{array}{l}T_{\text {eff }} \\
(\mathrm{K})\end{array}$ & $\begin{array}{c}V \sin i \\
\left(\mathrm{~km} \mathrm{~s}^{-1}\right)\end{array}$ & $\begin{array}{c}\langle\mathrm{RV}\rangle \\
\left(\mathrm{km} \mathrm{s}^{-1}\right)\end{array}$ & $\begin{array}{c}\mathrm{rms} \\
\left(\mathrm{km} \mathrm{s}^{-1}\right)\end{array}$ & $P\left(\chi_{\mathrm{RV}}^{2} \leqslant\right)$ \\
\hline GJ1001B & 4 & 398 & 1600 & $24.80 \pm 0.40$ & $32.84 \pm 0.17$ & 0.30 & 0.47 \\
\hline $2 \mathrm{M} 0015+35$ & 5 & 406 & 2200 & $10.23 \pm 2.55$ & $-37.35 \pm 0.16$ & 0.37 & 0.17 \\
\hline $2 \mathrm{M} 0036+18$ & 6 & 351 & 2000 & $35.12 \pm 0.57$ & $19.02 \pm 0.15$ & 0.26 & 0.05 \\
\hline $2 \mathrm{M} 0045+16$ & 6 & 351 & 2100 & $32.82 \pm 0.17$ & $3.29 \pm 0.17$ & 0.40 & 0.05 \\
\hline $2 \mathrm{M} 0141+18$ & 4 & 822 & 2200 & $<9.0$ & $24.65 \pm 0.13$ & 0.39 & 0.76 \\
\hline $2 \mathrm{M} 0144-07$ & 5 & 821 & 1600 & $23.03 \pm 0.45$ & $-2.55 \pm 0.10$ & 0.36 & 0.97 \\
\hline $2 \mathrm{M} 0213+44$ & 5 & 823 & 2200 & $12.89 \pm 2.34$ & $-23.47 \pm 0.11$ & 0.20 & 0.52 \\
\hline $2 \mathrm{M} 0227-16$ & 2 & 431 & 2100 & $<9.0$ & $48.58 \pm 0.15$ & 0.25 & 0.49 \\
\hline $2 \mathrm{M} 0228+25$ & 5 & 406 & 2100 & $31.19 \pm 0.81$ & $23.07 \pm 0.21$ & 0.29 & 0.13 \\
\hline $2 \mathrm{M} 0235-23$ & 6 & 823 & 2200 & $15.85 \pm 0.53$ & $15.38 \pm 0.11$ & 0.26 & 0.71 \\
\hline $2 \mathrm{M} 0251-03$ & 3 & 351 & 2100 & $21.76 \pm 0.38$ & $1.26 \pm 0.13$ & 0.31 & 0.80 \\
\hline $2 \mathrm{M} 0306-36$ & 2 & 358 & 1900 & $21.44 \pm 0.26$ & $11.44 \pm 0.19$ & 0.18 & 0.43 \\
\hline $2 \mathrm{M} 0320-04$ & 16 & 1180 & 1900 & $16.71 \pm 0.53$ & $3.13 \pm 0.06$ & 5.32 & $>0.99$ \\
\hline $2 \mathrm{M} 0355+11$ & 2 & 322 & 1900 & $12.31 \pm 0.15$ & $11.92 \pm 0.22$ & 0.45 & 0.50 \\
\hline $2 \mathrm{M} 0500+03$ & 2 & 322 & 1600 & $9.65 \pm 0.36$ & $15.94 \pm 0.16$ & 0.09 & 0.26 \\
\hline $2 \mathrm{M} 0523-14$ & 5 & 821 & 2100 & $15.98 \pm 0.31$ & $12.21 \pm 0.09$ & 0.19 & 0.70 \\
\hline $2 \mathrm{M} 0539-00$ & 4 & 614 & 1600 & $32.30 \pm 0.75$ & $13.91 \pm 0.15$ & 0.38 & 0.83 \\
\hline $2 \mathrm{M} 0543+64$ & 2 & 57 & 2200 & $20.06 \pm 0.52$ & $18.64 \pm 0.23$ & 0.11 & 0.26 \\
\hline LSR0602+39 & 10 & 1029 & 2100 & $12.51 \pm 0.22$ & $7.94 \pm 0.05$ & 0.24 & $>0.99$ \\
\hline $2 \mathrm{M} 0632+83$ & 2 & 96 & 1900 & $9.90 \pm 0.40$ & $-26.00 \pm 0.24$ & 0.06 & 0.11 \\
\hline $2 \mathrm{M} 0652+47$ & 10 & 1030 & 1600 & $30.08 \pm 1.76$ & $-7.03 \pm 0.07$ & 0.21 & 0.33 \\
\hline $2 \mathrm{M} 0700+31$ & 6 & 670 & 2100 & $29.91 \pm 0.27$ & $-42.42 \pm 0.09$ & 0.25 & 0.77 \\
\hline $2 \mathrm{M} 0717+57$ & 2 & 1 & 2100 & $13.93 \pm 0.55$ & $-16.32 \pm 0.17$ & 0.19 & 0.54 \\
\hline $2 \mathrm{M} 0746+20$ & 10 & 1030 & 2100 & $32.72 \pm 0.56$ & $52.37 \pm 0.06$ & 0.59 & $>0.99$ \\
\hline $2 \mathrm{M} 0828-13$ & 5 & 357 & 2300 & $29.13 \pm 5.00$ & $25.85 \pm 0.08$ & 0.28 & 0.93 \\
\hline $2 \mathrm{M} 0835-08$ & 8 & 1028 & 2200 & $14.18 \pm 0.43$ & $29.89 \pm 0.06$ & 0.15 & 0.52 \\
\hline $2 \mathrm{M} 0847-15$ & 5 & 999 & 2200 & $23.29 \pm 0.32$ & $2.02 \pm 0.10$ & 0.08 & 0.03 \\
\hline 2M0911+74 & 2 & 362 & 2000 & $12.18 \pm 0.59$ & $-4.06 \pm 0.15$ & 0.28 & 0.79 \\
\hline $2 \mathrm{M} 0921-21$ & 3 & 680 & 2000 & $11.95 \pm 0.49$ & $80.53 \pm 0.11$ & 0.05 & 0.04 \\
\hline $2 \mathrm{M} 1022+58$ & 3 & 682 & 2200 & $11.81 \pm 0.21$ & $19.29 \pm 0.11$ & 0.10 & 0.08 \\
\hline $2 \mathrm{M} 1045-01$ & 4 & 677 & 2200 & $<9.0$ & $6.31 \pm 0.10$ & 0.22 & 0.77 \\
\hline 2M1048+01 & 7 & 1030 & 2100 & $10.36 \pm 0.25$ & $24.25 \pm 0.06$ & 0.22 & 0.91 \\
\hline $2 \mathrm{M} 1108+68$ & 5 & 999 & 2100 & $26.03 \pm 0.29$ & $-9.84 \pm 0.11$ & 0.29 & 0.49 \\
\hline $2 \mathrm{M} 1112+35$ & 6 & 2249 & 2100 & $28.65 \pm 1.01$ & $-4.28 \pm 0.13$ & 0.39 & 0.33 \\
\hline $2 \mathrm{M} 1155-37$ & 2 & 264 & 2200 & $13.61 \pm 0.31$ & $45.57 \pm 0.11$ & 0.27 & 0.73 \\
\hline $2 \mathrm{M} 1203+00$ & 3 & 678 & 1600 & $31.33 \pm 0.55$ & $-0.22 \pm 0.16$ & 0.57 & 0.88 \\
\hline $2 \mathrm{M} 1221+02$ & 3 & 681 & 2000 & $23.82 \pm 0.42$ & $-8.79 \pm 0.14$ & 0.32 & 0.49 \\
\hline $2 \mathrm{M} 1300+19$ & 5 & 1040 & 2200 & $12.83 \pm 2.10$ & $-17.60 \pm 0.12$ & 0.22 & 0.28 \\
\hline $2 \mathrm{M} 1305-25$ & 2 & 29 & 2100 & $68.88 \pm 2.60$ & $6.37 \pm 0.35$ & 0.05 & 0.06 \\
\hline $2 \mathrm{M} 1425-36$ & 2 & 303 & 2000 & $32.37 \pm 0.66$ & $5.37 \pm 0.25$ & 0.25 & 0.38 \\
\hline 2M1439+19 & 5 & 1069 & 2200 & $11.10 \pm 0.24$ & $-26.74 \pm 0.09$ & 0.17 & 0.43 \\
\hline $2 \mathrm{M} 1506+13$ & 3 & 853 & 2200 & $11.39 \pm 0.94$ & $-0.68 \pm 0.11$ & 0.12 & 0.28 \\
\hline $2 \mathrm{M} 1507-16$ & 9 & 2249 & 1600 & $21.27 \pm 1.86$ & $-39.85 \pm 0.05$ & 0.27 & $>0.99$ \\
\hline $2 \mathrm{M} 1515+48$ & 4 & 707 & 1600 & $12.52 \pm 1.65$ & $-29.97 \pm 0.11$ & 0.14 & 0.13 \\
\hline 2M1539-05 & 3 & 29 & 1800 & $40.09 \pm 0.76$ & $27.33 \pm 0.24$ & 0.48 & 0.52 \\
\hline $2 \mathrm{M} 1552+29$ & 4 & 1237 & 2000 & $18.91 \pm 0.57$ & $-18.43 \pm 0.11$ & 0.03 & 0.01 \\
\hline 2M1555-09 & 5 & 1233 & 2200 & $<9.0$ & $14.84 \pm 0.10$ & 0.25 & 0.46 \\
\hline LSR1610-0040 & 4 & 1541 & 1900 & $16.84 \pm 3.11$ & $-97.89 \pm 0.21$ & 5.41 & $>0.99$ \\
\hline $2 \mathrm{M} 1645-13$ & 6 & 1234 & 2200 & $9.31 \pm 0.27$ & $26.58 \pm 0.06$ & 0.15 & 0.51 \\
\hline $2 \mathrm{M} 1658+70$ & 5 & 2041 & 2200 & $12.26 \pm 0.76$ & $-25.60 \pm 0.12$ & 0.17 & 0.20 \\
\hline 2M1705-05 & 3 & 425 & 2200 & $27.67 \pm 0.32$ & $12.19 \pm 0.11$ & 0.11 & 0.18 \\
\hline $2 \mathrm{M} 1731+27$ & 3 & 526 & 2000 & $11.62 \pm 0.15$ & $-29.76 \pm 0.11$ & 0.34 & 0.92 \\
\hline $2 \mathrm{M} 1807+50$ & 4 & 528 & 2100 & $69.88 \pm 2.48$ & $-0.36 \pm 0.46$ & 1.14 & 0.45 \\
\hline $2 \mathrm{M} 1821+14$ & 2 & 162 & 2200 & $28.85 \pm 0.16$ & $9.78 \pm 0.16$ & 0.14 & 0.43 \\
\hline $2 \mathrm{M} 1854+84$ & 3 & 351 & 2000 & $10.23 \pm 0.13$ & $-2.93 \pm 0.17$ & 0.10 & 0.10 \\
\hline $2 \mathrm{M} 2036+10$ & 2 & 146 & 2100 & $67.11 \pm 1.52$ & $19.66 \pm 0.47$ & 1.59 & 0.86 \\
\hline 2M2057-02 & 3 & 351 & 2100 & $60.56 \pm 0.37$ & $-24.68 \pm 0.43$ & 0.61 & 0.48 \\
\hline $2 \mathrm{M} 2104-10$ & 6 & 748 & 2200 & $23.44 \pm 0.23$ & $-21.09 \pm 0.12$ & 0.39 & 0.58 \\
\hline $2 \mathrm{M} 2224-01$ & 8 & 750 & 1600 & $25.49 \pm 0.41$ & $-37.55 \pm 0.09$ & 0.19 & 0.09 \\
\hline
\end{tabular}

Notes. Measured properties of the L dwarfs in our sample. $T_{\text {eff }}$ is based on the best-fit synthetic template with log $g=5.0$ (cgs). The errors on $V \sin i$ are estimated from the scatter in fits to multiple observations. The errors on the systemic velocity $\langle\mathrm{RV}\rangle$ are also determined from the scatter in the fits to multiple observations. The value of $P\left(\chi_{\mathrm{RV}}^{2} \leqslant\right)$ is the probability of getting a smaller value of $\chi_{\mathrm{RV}}^{2}$ and assumes the null hypothesis of no intrinsic RV variations.

${ }^{\text {a }}$ For this object we used a template with $\log g=5.5$. 
Table 4

RV Measurements of 2M0320-04

\begin{tabular}{lrc}
\hline \hline HJD-2400000 & $\begin{array}{c}\mathrm{RV} \\
\left(\mathrm{km} \mathrm{s}^{-1}\right)\end{array}$ & $\begin{array}{c}\sigma \\
\left(\mathrm{km} \mathrm{s}^{-1}\right)\end{array}$ \\
\hline 52921.102 & 6.148 & 0.378 \\
52922.113 & 6.550 & 0.294 \\
52957.027 & 4.392 & 0.327 \\
53272.125 & -6.443 & 0.234 \\
53273.086 & -6.461 & 0.235 \\
53328.828 & -4.113 & 0.225 \\
53421.719 & 6.274 & 0.194 \\
53669.887 & 5.905 & 0.291 \\
53670.879 & 6.363 & 0.191 \\
53686.859 & 5.453 & 0.178 \\
53742.809 & -2.912 & 0.331 \\
53743.856 & -3.140 & 0.258 \\
53744.840 & -3.378 & 0.407 \\
54023.969 & -7.276 & 0.216 \\
54100.746 & 1.134 & 0.295 \\
54101.754 & 1.398 & 0.673 \\
\hline
\end{tabular}

Table 5

Orbital and System Parameters for 2M0320-04

\begin{tabular}{lc}
\hline \hline \multicolumn{1}{c}{ Parameter } & Value \\
\hline Period (day) & $246.9 \pm 0.52$ \\
$e$ & $0.067 \pm 0.015$ \\
$\omega\left(^{\circ}\right)$ & $167.2 \pm 18.7$ \\
$T_{0}(\mathrm{MJD})$ & $53529.9 \pm 11.8$ \\
$K_{1}\left(\mathrm{~km} \mathrm{~s}^{-1}\right)$ & $6.92 \pm 0.12$ \\
$\gamma\left(\mathrm{km} \mathrm{s}^{-1}\right)$ & $0.154 \pm 0.072$ \\
$M_{2} \sin i\left(M_{\odot}\right)$ & $0.2032\left(M_{1}+M_{2}\right)^{2 / 3} \pm 0.0007$ \\
$a_{1} \sin i(\mathrm{AU})$ & $0.157 \pm 0.003$ \\
\hline
\end{tabular}

Notes. Estimated orbital and system parameters for 2M0320-04. These results are consistent with the earlier estimates from Blake et al. (2008b).

are known binaries already in the literature $(2 \mathrm{M} 0746+20$, LSR1610-0040), one is a new binary identified in the early stages of this survey (2M0320-04), and one target exhibits a possible long-term RV trend (2M1507-16). LSR0602+39 exhibits statistically significant RV variations that show no evidence for periodicity or a long-term trend. This object is an $\mathrm{L}$ subdwarf discovered in the galactic plane by Salim et al. (2003) and, based on the detection of $\mathrm{Li}$ in the spectrum, is thought to be a brown dwarf. Possibly because of its low metallicity, our theoretical spectral templates are a comparatively poor fit for the observed spectrum of this object, particularly around the $\mathrm{CO}$ bandhead, and as a result our theoretical error estimates are possibly underestimated. We also compared our RVs to the few that exist in the literature, including those from Blake et al. (2007), to look for any additional evidence of long-term trends. While we found overall good agreement with our measurements, we note that the RV for Kelu-1 (2M1305-25) reported by Basri et al. (2000) from observations in 1997 differs by about $10 \mathrm{~km} \mathrm{~s}^{-1}$ from our measurement in 2003. Kelu-1 is a binary, or possibly a triple, system with an estimated orbital period of 38 years (Gelino et al. 2006; Stumpf et al. 2008), with an expected RV semi-amplitude of 3-4 $\mathrm{km} \mathrm{s}^{-1}$. We found no such offset for the other objects observed in common with Basri et al. (2000).

Measuring the reflex motion of the primary component of a single-lined binary (SB1) system, $K_{1}$, allows us to measure the mass function, a transcendental equation that involves $M_{1}, M_{2}$,

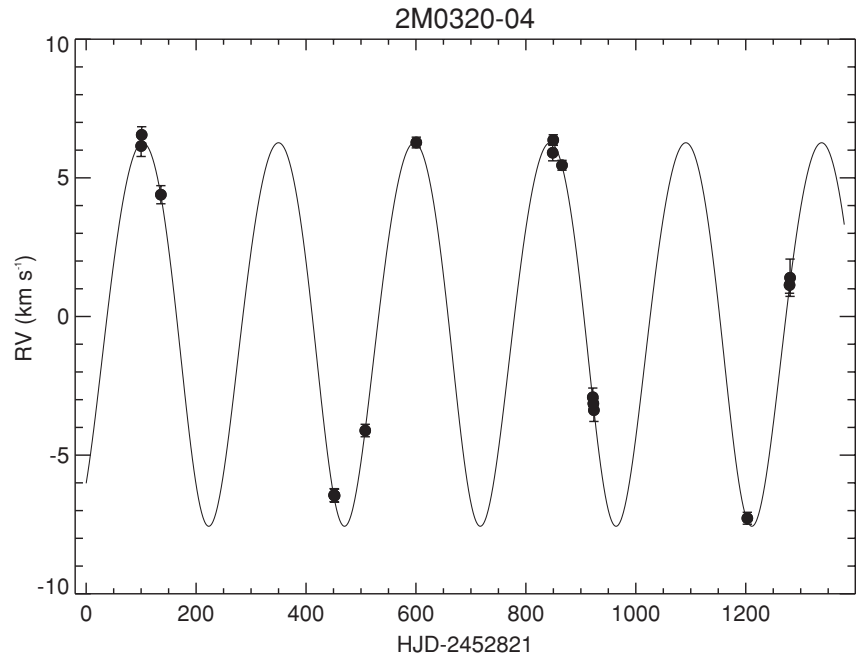

Figure 10. Best-fit orbital solution for 2M0320-04. The scatter of the data about the model is $135 \mathrm{~m} \mathrm{~s}^{-1}$.

and $\sin i$ :

$f(m)=\frac{M_{2}^{3} \sin ^{3} i}{\left(M_{1}+M_{2}\right)^{2}}=\left(1.0361 \times 10^{-7}\right)\left(1-e^{2}\right)^{3 / 2} K_{1}^{3} P M_{\odot}$,

where $P$ is in days and $K_{1}$ is in $\mathrm{km} \mathrm{s}^{-1}$. In a double-lined spectroscopic binary system (SB2) the measurements of $K_{1}$ and $K_{2}$ can be combined to directly estimate the mass ratio $q=M_{2} / M_{1}$. Additional observations, such as astrometric observations of the binary orbit, are required to determine $\sin i$ and to measure any of these quantities independently. We used a nonlinear Levenberg-Marquardt fitting scheme (Markwardt 2009) to fit the six Keplerian orbital parameters to our data and analyze the RV variations of four of our targets. The six orbital parameters we fit are $K_{1}$, the RV semi-amplitude of the primary; $\gamma$, the systemic velocity of the center of mass of the system; $\omega$, the longitude of periastron; $e$, the eccentricity; $T_{0}$, the time of periastron passage; and $P$, the orbital period.

\section{1. $2 M 0320-04$}

We discovered the SB1 spectroscopic binary system 2M0320-04 early in our survey (Blake et al. 2008b) and its binarity was suggested independently using spectral fitting methods by Burgasser et al. (2008). With a relatively short period $(P=246.9 \pm 0.52 \mathrm{~d})$ and large $\mathrm{RV}$ semi-amplitude $\left(K=6.92 \pm 0.12 \mathrm{~km} \mathrm{~s}^{-1}\right)$, this system was easily detected in our NIRSPEC data and was observed on 16 epochs during the course of our survey. We fit the spectroscopic orbit using $\mathrm{RV}$ measurements that are improved over those in Blake et al. (2008b) and found consistent results with a scatter about the fit of $135 \mathrm{~m} \mathrm{~s}^{-1}$, improved from the $350 \mathrm{~m} \mathrm{~s}^{-1}$ scatter found using the analysis pipeline described in Blake et al. (2008b). The new RV measurements and the orbital solution are shown in Tables 4 and 5 and Figure 10. Based on the spectral analysis of Burgasser et al. (2008), this system is thought to be composed of a late-M dwarf and an early-T dwarf with masses $M_{1} \simeq 0.08 M_{\odot}$ and $M_{2} \simeq 0.054 M_{\odot}$. As a single-lined system, our spectroscopic orbit results in a mass function and without additional observations to determine $\sin i$, the individual masses, or their ratios, cannot be directly measured. While this system is expected to be too narrowly separated ( $\theta \sim 17$ mas) to be resolved with AO systems on current telescopes, with more sensitive observations the 
Table 6

RV Measurements of LSR1610-0040

\begin{tabular}{lll}
\hline \hline HJD-2400000 & \multicolumn{1}{c}{$\begin{array}{c}\text { RV } \\
\left(\mathrm{km} \mathrm{s}^{-1}\right)\end{array}$} & $\begin{array}{c}\sigma \\
\left(\mathrm{km} \mathrm{s}^{-1}\right)\end{array}$ \\
\hline 53421.176 & -95.041 & 0.436 \\
$53431.455^{\mathrm{a}}$ & -95 & 1 \\
53597.766 & -97.629 & 0.266 \\
$53800.835^{\mathrm{b}}$ & -108.1 & 1.6 \\
53948.773 & -95.828 & 0.726 \\
54962.938 & -106.482 & 0.686
\end{tabular}

Notes.

a Basri \& Reiners (2006).

${ }^{b}$ Dahn et al. (2008).

Table 7

Orbital and System Parameters for LSR16010-0040

\begin{tabular}{lc}
\hline \hline \multicolumn{1}{c}{ Parameter } & Value \\
\hline Period (day; fixed) & $607.1 \pm 4.34$ \\
e (fixed) & $0.444 \pm 0.017$ \\
$i\left(^{\circ} ;\right.$ fixed $)$ & $83.2 \pm 1.0$ \\
$\omega\left(^{\circ} ;\right.$ fixed $)$ & $151.4 \pm 4.6$ \\
$T_{0}(\mathrm{MJD})$ & $53680.2 \pm 8.2$ \\
$K_{1}\left(\mathrm{~km} \mathrm{~s}^{-1}\right)$ & $8.02 \pm 0.90$ \\
$\gamma\left(\mathrm{km} \mathrm{s}^{-1}\right)$ & $-99.49 \pm 0.41$ \\
$M_{2}^{3}\left(M_{\odot}\right)$ & $0.025\left(M_{I}+M_{2}\right)^{2} \pm 0.008$ \\
$a_{1}(\mathrm{AU})$ & $0.404 \pm 0.042$ \\
\hline
\end{tabular}

Notes. Estimated orbital and system parameters for LSR1610-0040 assuming the astrometric orbital parameters from Dahn et al. (2008). Using four NIRSPEC measurements and one other from the literature we fit for the time of periastron passage, $T_{0}$, the systemic velocity, $\gamma$, and the RV semi-amplitude, $K_{1}$.

spectral lines of the secondary should be detected. In that case, the ratio of the masses and the ratio of the brightnesses can be determined using a technique like TODCOR (Mazeh \& Zucker 1994). Such measurements would allow for some of the first direct tests of coeval UCDs at field $(t>1 \mathrm{Gyr})$ ages, providing important constraints on theoretical models. The flux ratio at $K$ band, where our NIRSPEC data were taken, is expected to be between $100: 1$ and $5: 1$, and thus may be below our detection limit. The estimated mass ratio of $0.6 \lesssim M_{2} / M_{1} \lesssim 0.8$ (Burgasser \& Blake 2009) would indicate a maximum velocity separation between the two components of $15 \mathrm{~km} \mathrm{~s}^{-1} \lesssim \Delta V \lesssim$ $18 \mathrm{~km} \mathrm{~s}^{-1}$, potentially resolvable with NIRSPEC.

In order to search for the spectral lines of the secondary, we created an empirical spectral template for 2M0320-04 following the same method described in Section 5.1 with the additional step of correcting the wavelength solutions for the SB1 orbit determined above. We found no evidence for temporal variations in the residuals of the fits. Using an empirical template to fit the NIRSPEC observations of the rapidly rotating ( $V \sin i=30.1 \mathrm{~km} \mathrm{~s}^{-1}$ ) object $2 \mathrm{M} 0652+47$ we found that the residuals to the fits were at the level of $1 \%$. For rapidly rotating objects any changes in the spectrograph LSF have a small impact on the empirical template generation. This is not the case for 2M0320-04, where $V \sin i=16.7 \mathrm{~km} \mathrm{~s}^{-1}$ is slightly larger than the width of the LSF. In order to quantify the impact of this effect, we studied fits with an empirical template for the NIRSPEC observations of $2 \mathrm{M} 0835-08\left(V \sin i=14.18 \mathrm{~km} \mathrm{~s}^{-1}\right)$. In this case, we found that fit residuals using the empirical template were as large as $5 \%$ in the cores of CO features. In the future it

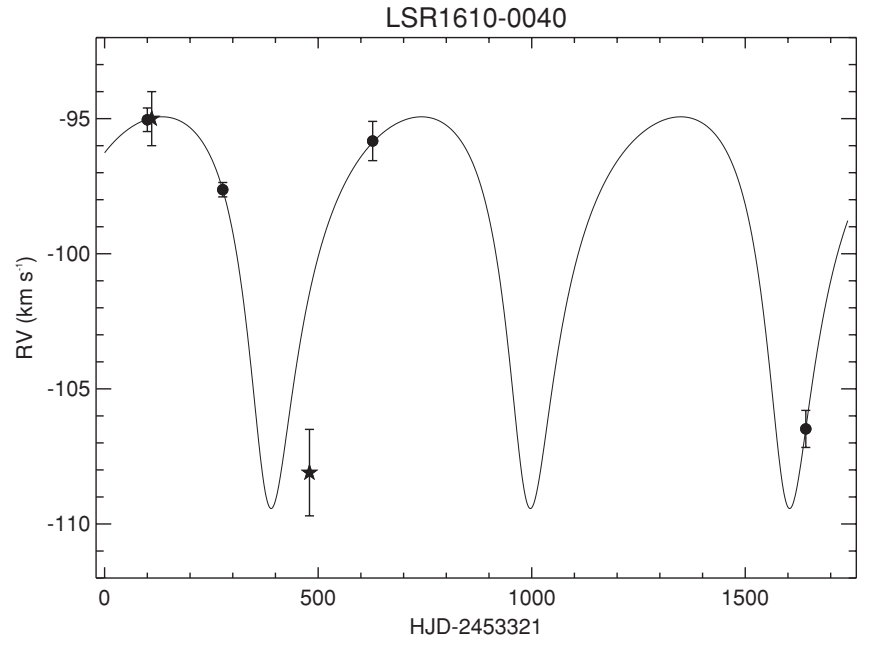

Figure 11. Orbital solution for the subdwarf binary LSR1610-0040 (solid line) assuming $P, e$, and $\omega$ from Dahn et al. (2008) and fitting for $\gamma, T_{0}$, and $K_{1}$. Circles are our measurements, stars are from the literature (Dahn et al. 2008; Basri \& Reiners 2006). The single point from Dahn et al. (2008) is incompatible with our RV measurements and the astrometric orbital solution and is excluded from our analysis.

may be possible to create an empirical template by deconvolving the individual spectra with a more realistic estimate of the LSF, increasing our sensitivity to faint companions.

\section{2. $L S R 1610-0040$}

LSR1610-0040 (LSR1610) is a known binary with a lowmass primary and an orbital solution based on fits to the astrometric motion of the center of light of the system (Dahn et al. 2008). We combined our four measurements with two others from the literature (Reiners \& Basri 2006; Dahn et al. 2008) and found good agreement between our RV measurements and the orbital parameters determined from the astrometric orbit alone. The single measurements from Dahn et al. (2008) derived from fitting individual atomic resonance lines in the optical spectrum deviates significantly from the expected spectroscopic orbit and is excluded from the following analysis. The single measurement from Reiners \& Basri (2006), also at optical wavelengths, is nearly coincident with one of our RV measurements and the two agree within $1 \sigma$. We performed a Monte Carlo simulation to estimate the parameters of the spectroscopic orbit by varying $P, e, i$, and $\omega$ according to the quoted errors in Dahn et al. (2008) while also varying the RV measurements according to their quoted errors and fitting for $K_{1}, T_{0}$, and $\gamma$. The RV measurements and best-fit spectroscopic orbital orbital parameters are given in Tables 6 and 7 and the orbital solution is shown in Figure 11 . The primary of the system is expected to be very low in mass and assuming that the flux of the secondary is negligible. Dahn et al. (2008) estimate $0.09 M_{\odot}<M_{1}<0.098 M_{\odot}$ using the mass-luminosity relation (MLR) from Delfosse et al. (2000).

The orbital motion of the center of light from Dahn et al. (2008), denoted $\alpha$, is related to the physical properties of the system as

$$
\alpha=a_{1}\left[1-\beta_{I}\left(M_{1}+M_{2}\right) / M_{2}\right]=0.276 \mathrm{AU} .
$$

Based on the parallax from Dahn et al. (2008) the distance modulus for LSR1610 is $m-M=2.54 \pm 0.018$ and $\alpha$ is known in absolute units. Combining the astrometric orbital period, eccentricity, and inclination with the RV semi-amplitude, 


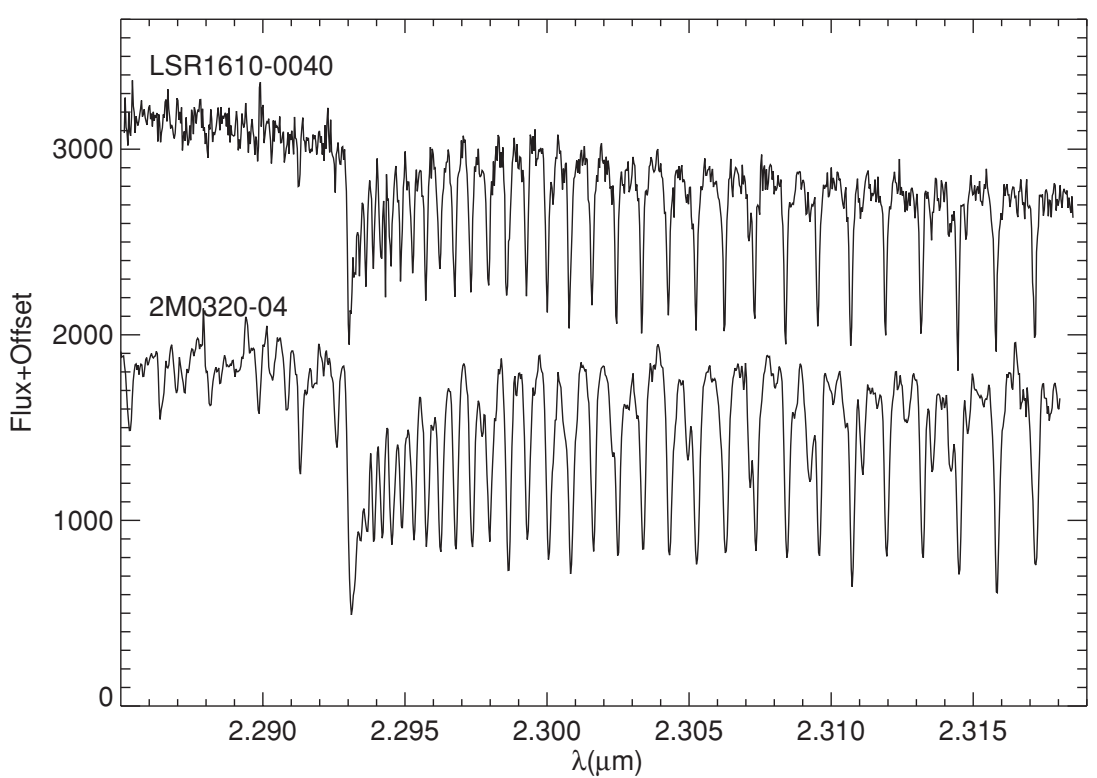

Figure 12. NIRSPEC spectrum of LSR1610-0040 compared to the spectrum of the M8/L0 dwarf 2M0320-04. While the overall structure of the CO bandhead is similar, the lack of strong absorption features in LSR1610 blueward of the bandhead is possible evidence for low metallicity.

Table 8

LSR1610

\begin{tabular}{lcc}
\hline \hline$M_{1}$ & $M_{2}$ & $\beta_{I}$ \\
$\left(M_{\odot}\right)$ & $\left(M_{\odot}\right)$ & \\
\hline 0.20 & 0.144 & 0.138 \\
0.15 & 0.124 & 0.150 \\
0.10 & 0.10 & 0.166 \\
0.095 & 0.098 & 0.168 \\
0.090 & 0.096 & 0.170 \\
0.085 & 0.093 & 0.172 \\
0.08 & 0.900 & 0.175 \\
0.075 & 0.087 & 0.177 \\
0.07 & 0.085 & 0.181 \\
\hline
\end{tabular}

Notes. System parameters from the combined analysis of the astrometric and spectroscopic orbits for a range of $M_{1}$. The mass of the primary uniquely determines the mass of the secondary, $M_{2}$, and the $I$-band flux ratio, $\beta_{I}=l_{2} /\left(l_{1}+12\right)$.

$K_{1}$, directly determines the semi-major axis $a_{1}$. The spectroscopic mass function (Equation (5)) and the relation for $\alpha$ are two equations in three unknowns $\left(M_{1}, M_{2}\right.$, and $\left.\beta_{I}\right)$ since the spectroscopic mass function combined with the known inclination determines $M_{2}$ as a function of $M_{1}$. These relations together determine the $I$-band light ratio $\beta_{I}=\left(1+10^{0.4 \Delta I}\right)^{-1}$ as a function of $M_{1}$. In Table 8, we list $M_{2}$ and $\beta_{I}$ derived from the combination of the astrometric and spectroscopic orbits for a range of $M_{1}$.

LSR1610 has been described as having "schizophrenic" spectral properties and is in many ways an enigmatic object (Cushing \& Vacca 2006; Reiners \& Basri 2006). The spectral features in the optical and NIR are indicative of a mildly metal poor mid-M dwarf with a number of unusual atomic absorption features. In Figure 12, we compare our high-resolution $K$-band spectra of this object to the spectrum of the M8/L0 dwarf 2M0320-04. While the overall structure of the CO bandhead and the strengths of the individual CO features is quite similar in these objects, we interpret the lack of absorption features blueward of the bandhead as possible evidence for low metallicity. Dahn et al. (2008) found that the combined light

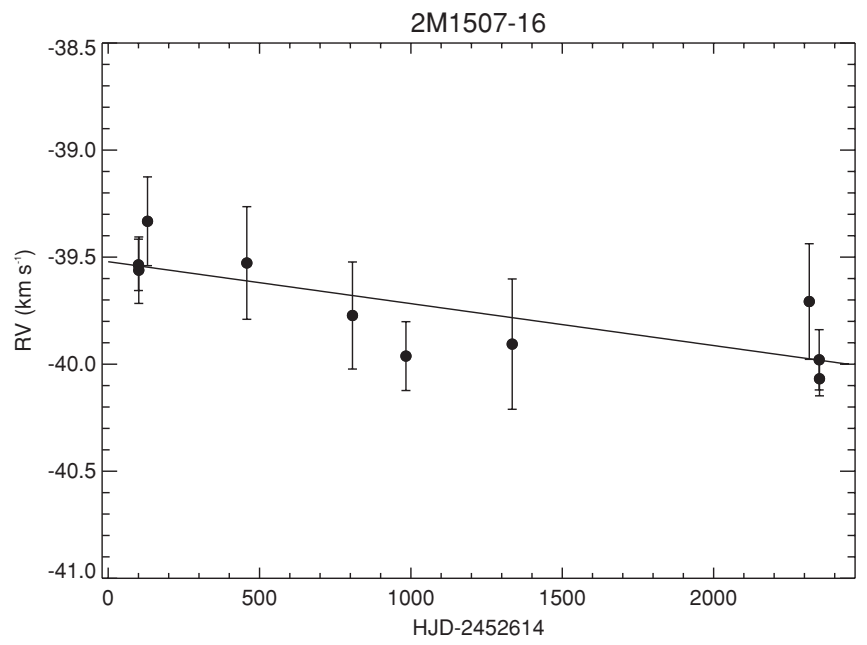

Figure 13. RV measurements for $2 \mathrm{M} 1507-16$. The solid line is a linear RV trend that may be indicative of a very long period orbit.

of the system falls very near the locus of low-mass stars on color-magnitude diagrams (CMDs), both $M_{V}$ versus $V-I$ and $M_{K_{s}}$ versus $I-K_{s}$, meaning that it has roughly the expected luminosity and colors for an $\mathrm{M}$ dwarf. At the same time, they attribute a $B$ flux that is significantly suppressed to metal pollution by accretion of material from a hypothetical AGB star that could also be responsible for prominent $\mathrm{Al}$ lines in the NIR spectrum. Here, we summarize what we know about this system and attempt to provide some possible explanations of the observed properties.

1. If we assume that the primary is indeed low in mass, say $0.075 M_{\odot}<M_{1}<0.2 M_{\odot}$, then $0.138<\beta_{I}<0.177$. If we assume an average value of $\beta_{I}=\beta_{K_{s}}=0.16 \pm 0.02$ then the absolute magnitude of LSR1610A is $M_{K_{s}}=$ $9.67 \pm 0.031 \mathrm{mag}$. Our analysis constrains the mass ratio ( $\left.q=M_{2} / M_{1}\right)$ of the system to be $0.83<q<1.4$ for $0.075 M_{\odot}<M_{1}<0.2 M_{\odot}$.

2. MLRs are thought to be least sensitive to metallicity at NIR wavelengths. The updated $K$-band MLR from Xia 
et al. (2008) would indicate a mass for LSR1610A of $M_{1}=0.103 M_{\odot}$ after correcting for the light of LSR1610B, somewhat larger than the estimate based on the Delfosse et al. (2000) relations of $M_{1}=0.088 M_{\odot}$, though $M_{K}=$ 9.67 falls outside the range over which the Delfosse et al. (2000) relation is formally defined.

3. Schilbach et al. (2009) found good general agreement between theoretical isochrones for old, low-metallicity stars from Baraffe et al. (1998) and the absolute magnitudes of 10 low-mass subdwarfs with measured parallaxes. Using slightly metal poor Lyon models $([\mathrm{M} / \mathrm{H}]=-0.5, t=$ $10 \mathrm{Gyr}$ ), we estimated the mass of LSR1601A to be $M_{1}=$ $0.095 M_{\odot}$ and found a similar mass if the system is 1,5 , or 10 Gyr. These models also agree with the observed $J-K_{s}$ color of the combined light of this system. This object has a very large space velocity $\left(V_{\mathrm{tot}}=243.3 \pm 2.0 \mathrm{~km} \mathrm{~s}^{-1}\right.$ ), and based on the kinematic arguments in Section 7 we expect that this system is part of an older, thick disk population, making it unlikely that it is in fact very young.

Based on the available observational evidence it remains difficult to explain LSR1610. From the empirical MLRs and theoretical isochrones it seems that LSR1610A is a low-mass star with $M_{1} \simeq 0.1 M_{\odot}$. For the mass range $M_{1}<0.1 M_{\odot}$ the spectroscopic mass function requires that $M_{1} \leqslant M_{2}$, a somewhat implausible scenario if $\beta_{I}=0.16$ since the theoretical expectation is that the more massive component should be more luminous across the optical and infrared. The problem is alleviated somewhat if in fact LSR1610A is more massive than expected. Based on the available observational evidence, we propose the following possible scenarios for LSR1610.

1. The distance of LSR1610 could be underestimated. This would increase the mass estimate based on the isochrones and the MLRs and result in $M_{1}>M_{2}$. For example, if $M_{1}=0.2 M_{\odot}$ then $M_{1}>M_{2}$ and the flux difference $\Delta I=1.99 \mathrm{mag}$ is consistent with theoretical models. This may seem an implausible explanation since Dahn et al. (2008) and Schilbach et al. (2009) find consistent parallaxes.

2. LSR1610B is a very unusual object. In principle, LSR1610B could be an old compact object but would have to be very faint in $B\left(M_{B} \sim 22.4\right)$ yet relatively bright in the $I$ band $\left(M_{I} \sim 14.3, B-I \sim 8\right)$. Based on the known properties of low-mass white dwarfs (Kilic et al. 2007), we deem this scenario unlikely. Dahn et al. (2008) reached a similar conclusion.

It is possible that in the near future this system could be resolved with aperture masking interferometry (e.g., Ireland et al. 2008), resulting in a direct measurement of the masses of both components. We also carried out an empirical template analysis for this system, correcting the wavelength solutions for both the barycentric motion and the SB1 orbital solution. The expected that velocity separation is rather large for this system $\left(\Delta \mathrm{RV} \sim 25 \mathrm{~km} \mathrm{~s}^{-1}\right)$ and the flux ratio in $K$ band is expected to be 5:1 making LSR1610 an excellent candidate for a double-lined system. Unfortunately, we have only one epoch of observations where the orbital phase is favorable for resolving the lines of the secondary and those spectra were obtained under poor observing conditions and are of relatively low $\mathrm{S} / \mathrm{N}$. We found no evidence for the lines of the secondary in the residuals of the fits at this epoch using an empirical template generated using the technique described in Section 5.1.
Table 9

RV Measurements of 2M1507-16

\begin{tabular}{lcc}
\hline \hline HJD-2400000 & $\begin{array}{c}\mathrm{RV} \\
\left(\mathrm{km} \mathrm{s}^{-1}\right)\end{array}$ & $\begin{array}{c}\sigma \\
\left(\mathrm{km} \mathrm{s}^{-1}\right)\end{array}$ \\
\hline 52714.066 & -39.536 & 0.120 \\
52715.105 & -39.561 & 0.155 \\
52744.043 & -39.333 & 0.207 \\
53072.102 & -39.527 & 0.263 \\
53421.086 & -39.773 & 0.250 \\
53597.730 & -39.962 & 0.161 \\
53948.742 & -39.906 & 0.304 \\
54930.055 & -39.707 & 0.270 \\
54962.895 & -39.979 & 0.141 \\
54963.895 & -40.068 & 0.080
\end{tabular}

\section{3. $2 M 1507-16$}

This object is a very nearby mid-L dwarf with a parallax of $136.4 \pm 0.6$ mas (Dahn et al. 2002). Our initial RV measurements of this object exhibited a statistically significant RV trend. We obtained additional NIRSPEC measurements under cloudy conditions in 2009 April and May, resulting in a total time baseline of more than six years, as shown in Figure 13. The amplitude of the RV variation is close to the limits of detection with our current technique, but we rule out constant RV $\left[P\left(\chi_{\mathrm{RV}}^{2} \leqslant\right)>0.999\right]$. The measurements, given in Table 9, are well fit by a linear RV trend with a slope of $-72 \pm 23 \mathrm{~m} \mathrm{~s}^{-1}$ per year. Using a Monte Carlo simulation, we estimated the false alarm probability of observing a slope $|d \mathrm{RV} / D t|>72 \mathrm{~m} \mathrm{~s}^{-1}$ per year to be $2.2 \%$. This linear trend is also consistent with a single RV measurement from Bailer-Jones (2004) obtained in 2000, though this optical measurement has a very large error bar $\left(-39.3 \pm 1.5 \mathrm{~km} \mathrm{~s}^{-1}\right.$ near HJD 2451661). Basri \& Reiners (2006) report a velocity difference between 2000 and 2004 of $2.5 \mathrm{~km} \mathrm{~s}^{-1}$ for this object, which is incompatible with the velocity trend seen in our data, though we note that these measurements also have large error bars. We calculated the secular RV acceleration $(d \mathrm{RV} / d t)$ for this object following Kürster et al. (2003) and estimated the maximum amplitude of this effect to be less than $0.6 \mathrm{~m} \mathrm{~s}^{-1} \mathrm{yr}^{-1}$, far smaller than the observed trend. The trend seen in our NIRSPEC observations could be indicative of a very long period system with $P>5000$ days, but we note that within our sub-sample of 46 objects observed on three or more epochs we may expect to observe at least one long-term slope with a false-alarm probability of $2.2 \%$

The mass of the L5 primary is poorly constrained based on photometry alone, but comparing absolute magnitudes and the temperature $\left(M_{K}=12.07, M_{\mathrm{bol}}=15.32\right.$, and $T_{\text {eff }}=1703 \pm 60$ from Dahn et al. 2002) to models from Baraffe et al. (2003) and Chabrier et al. (2000), the minimum mass is expected to be in the range $0.06 M_{\odot}<M_{1}<0.07 M_{\odot}$ depending on the age of the system, assuming (based on the object's kinematics) $t>1 \mathrm{Gyr}$. Based on the lack of Li absorption, Reid et al. (2000) determined that this $\mathrm{L}$ dwarf has a minimum mass of $0.06 M_{\odot}$. If we assume that the binary has near equal mass components, as is observed to be the case for many low-mass binaries (Allen 2007), with $M_{1}=M_{2}=0.065 M_{\odot}$, then the mean orbital separation for a circular orbit would be $a>2.9$ AU if $P>5000$ days. At a distance of $7.3 \mathrm{pc}$ this corresponds to an angular separation of $\theta>0$.'4. Unless the system is close to edge-on and observed at a very unfavorable phase, then such a binary would have been 
readily resolved in the high-resolution imaging observations presented by Bouy et al. (2003) and Reid et al. (2006) who found no luminous ( $\Delta m<5 \mathrm{mag}$ ) companions to $2 \mathrm{M} 1507-16$ over a range of separations $00^{\prime} .05<\theta<1^{\prime \prime}$. Approximating the detection limit for faint companions from Reid et al. (2006) as $\Delta M_{J}<5$ and assuming the 5 Gyr models from Baraffe et al. (2003) for the secondary and 5 Gyr models from Chabrier et al. (2000) for the primary then the non-detection constrains the mass ratio of the system to be $q<0.15$. It is also possible that the period of the system is much longer than 5000 days, resulting in an angular separation too large to be detected in the narrow fields of view of high-resolution imaging surveys. Using NIR imaging data obtained in 2005 and described in Blake et al. (2008a) we detect only one source in an annulus of $5^{\prime \prime}$ to $20^{\prime \prime}$ down to limiting magnitudes of $J \sim 18.2$ and $K \sim 16.3$. This source is also visible in archival 2MASS observations from 1998 and does not share the large $\left(0^{\prime} .9\right.$ year $^{-1}$; Dahn et al. 2002) proper motion of 2M1507-16.

\section{4. $2 M 0746+20$}

The orbital motion of the two components of this tight $(\theta \sim 150$ mas) binary system has been directly observed with high-resolution imaging, resulting in the measurement of the sum of the masses and the first dynamical mass estimate for an L dwarf (Bouy et al. 2004). Based on high spatial resolution observations with the Hubble Space Telescope, VLT, and Gemini, the orbital period of this system is estimated to be $P=3850.9_{-767}^{+904}$ days, so our NIRSPEC observations, shown in Figure 14, span a considerable fraction of a period. The fit to the astrometric orbit determines the sum of the masses, $M_{1}+M_{2}=$ $0.146_{-0.006}^{+0.016} M_{\odot}$, and the semi-major axis $a=2.53_{-0.28}^{+0.37}$ AU. The components of this system are resolved and the flux ratio of the components is measured to be 1.6:1 at $K$ band. Based on the orbital parameters determined in Bouy et al. (2004) and the mass estimates from Gizis \& Reid (2006), the expected RV separation at periastron passage is $K_{1}+K_{2} \simeq 7 \mathrm{~km} \mathrm{~s}^{-1}$. Using NIRSPEC with AO, Konopacky et al. (2010) resolved this system and obtained RV measurements for both components. Combining these measurements with the astrometric orbit yields direct measurements of the masses of both components, though for the case of $2 \mathrm{M} 0746+20$ the errors on the mass estimates are relatively large.

While the flux ratio of the system is favorable for detecting the spectral lines of the secondary, the velocity separation is small compared to the NIRSPEC resolution, particularly for observations that do not coincide with the periastron passage in 2003. In this case, where both components are of similar brightness, are expected to have similar spectral features, and are not resolved in velocity, we are effectively measuring the $\mathrm{RV}$ of the combined light of the system. If the components have exactly equal masses, spectral features, and luminosities, we would observe no RV variations at all. As a result of this combined light effect we do not necessarily expect to measure the true RV of $2 \mathrm{M} 0746+20 \mathrm{~A}$ using our technique. Using the orbital parameters from the astrometric orbit from Bouy et al. (2004) we fit for $K_{1}$ and $\gamma$ assuming that the light from $2 \mathrm{M} 0746+20 \mathrm{~B}$ has a negligible impact on the measured RV and found $K_{1}=0.9 \pm 0.18 \mathrm{~km} \mathrm{~s}^{-1}$ and $\gamma=53.1 \pm 0.28 \mathrm{~km} \mathrm{~s}^{-1}$. The resulting RV orbit is shown in Figure 14. We emphasize that these orbital parameters are very likely biased by the influence of $2 \mathrm{M} 0746+20 \mathrm{~B}$, in particular our estimate of $K_{1}$ is expected to be too low. We carried out a simulation to assess the impact of a marginally resolved secondary by fitting

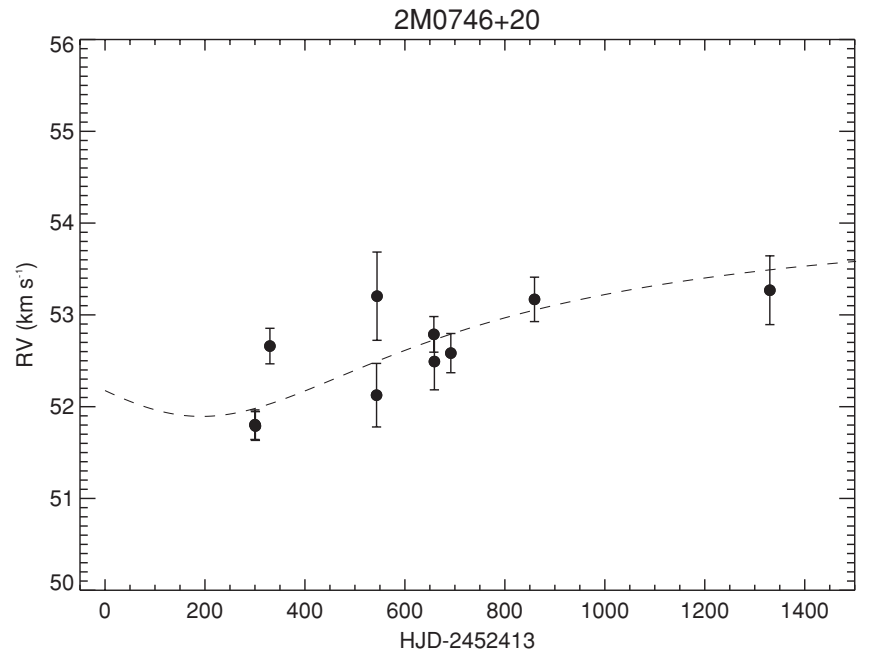

Figure 14. RV measurements of $2 \mathrm{M} 0746+20$. The dashed line is the orbit of $2 \mathrm{M} 0746+20 \mathrm{~A}$ based on the astrometric observations of Bouy et al. (2004) after fitting for $K_{1}$ and $\gamma$ with our RV measurements. The spectra of the two components of this system are not resolved in our NIRSPEC observations and so we measure the effective RV of the combined light.

mock spectra that contained light from a secondary with a flux ratio of $F_{1} / F_{2}=1.5$ and velocity offsets of between $2 \mathrm{~km} \mathrm{~s}^{-1}<\Delta \mathrm{RV}<10 \mathrm{~km} \mathrm{~s}^{-1}$ from the primary. We found that such companions significantly influenced the resulting $\mathrm{RV}$ measurement from fitting the combined light, $\mathrm{RV}_{C}$, and that $\left|\mathrm{RV}-\mathrm{RV}_{C}\right| \approx \Delta \mathrm{RV}\left(F_{2} / F_{1}\right)$, meaning that our $\mathrm{RV}$ measurements near periastron passage could be biased by up to a few $\mathrm{km} \mathrm{s}^{-1}$.

\section{ROTATIONAL AND SPACE VELOCITIES}

Our observations can also be used to study the statistics of the rotational and space velocities of UCDs. The rotational velocities of brown dwarfs and low-mass stars are important for understanding the angular momentum history of these objects. Measurements of a large number of UCD $V \sin i$ values exist in the literature, though the measurements were made using techniques different from the one employed here. Reiners \& Basri (2008) estimated rotational velocities by fitting the rotationally broadened $\mathrm{FeH}$ stellar absorption features near $1 \mu \mathrm{m}$ using data from HIRES at Keck I and UVES at VLT. These optical data are of higher resolution than our NIRSPEC data resulting in $V \sin i$ sensitivity down to $3 \mathrm{~km} \mathrm{~s}^{-1}$. Both Bailer-Jones (2004), using VLT and the UVES spectrograph, and Zapatero Osorio et al. (2007), using Keck and NIRSPEC, follow the traditional technique of cross-correlating their spectra with a slowly rotating template and estimating $V \sin i$ from the width of the resulting cross-correlation function. As shown in Figure 15, we find excellent overall agreement between our $V \sin i$ estimates and the values reported in the literature, including those in Blake et al. (2007).

Reiners \& Basri (2008) explored possible patterns in the rotation of $\mathrm{M}$ and $\mathrm{L}$ dwarfs as a function of spectral type in an effort to better understand the evolution of the angular momentum of UCDs as they age. The fact that both young and old $\mathrm{L}$ dwarfs are often rapid rotators indicates that their rotation differs from that of Sun-like stars. More massive stars lose angular momentum through solar winds, slowing as they age, but older field L dwarfs still appear to be relatively rapid rotators compared to Sun-like stars of similar ages. 


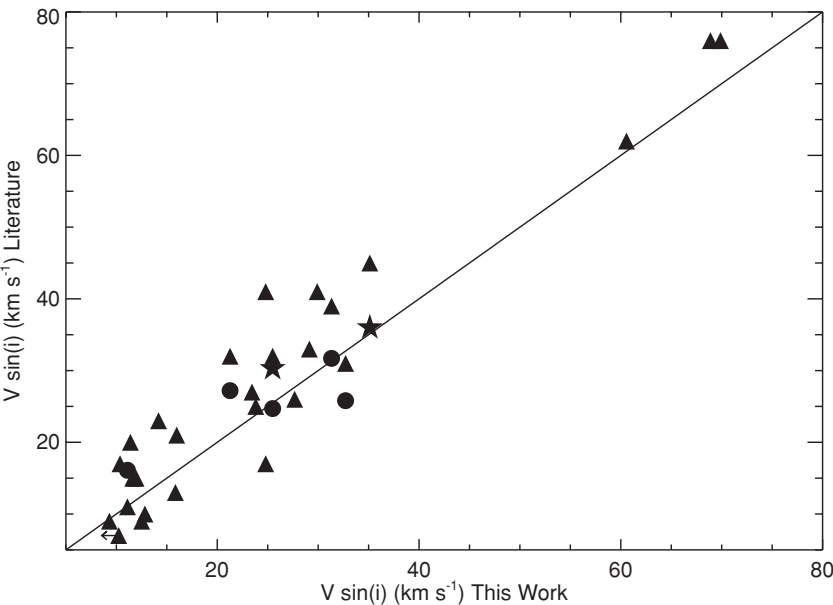

Figure 15. Our $V \sin i$ measurements compared to those from the literature Triangles are from Reiners \& Basri (2008), circles are from Bailer-Jones (2004), and stars are from Zapatero Osorio et al. (2007). While there is significant scatter in the $V \sin i$ estimates we find overall good agreement between our measurements and those from the literature.

Reiners \& Basri (2008) propose a braking mechanism that decreases in efficiency at the cooler atmospheric temperatures of UCDs and is very inefficient in mid- to late-L dwarfs, where the deceleration timescales become comparable to the age of the universe. At the cool temperatures of UCDs the stellar atmospheres are largely neutral, with few ions, reducing the strength of the coupling between the magnetic fields and the atmosphere and therefore reducing the strength of the braking mechanism. This model explains the lack of $\mathrm{L}$ dwarfs later than L3 found by Reiners \& Basri (2008) to rotate slower than $V \sin i=20 \mathrm{~km} \mathrm{~s}^{-1}$. In Figure 16, we show our projected rotational velocities against spectral type as determined from optical diagnostics, the same spectral types used by Reiners \& Basri (2008), along with other measurements from the literature. We also see some evidence for increasing $V \sin i$ for objects with spectral types later than L3. We do, however, find four mid-L dwarfs that appear to be very slow rotators with $V \sin i<20 \mathrm{~km} \mathrm{~s}^{-1}: 2 \mathrm{M} 0355+11,2 \mathrm{M} 0500+03,2 \mathrm{M} 0835-08$, and $2 \mathrm{M} 1515+48$. The spectra of these L dwarfs, shown in Figure 17, exhibit sharp, narrow CO features indicative of slow rotation and their spectra are relatively well fit by our theoretical UCD models. The L6 dwarf 2M1515+48 also has a very large space velocity, a potential indicator of old age. The Reiners \& Basri (2008) sample includes approximately $15 \mathrm{~L}$ dwarfs with spectral types later than L3, comparable to our number. It is possible that these results are entirely consistent and that our slow rotators are simply observed close to pole-on. Comparing our $V \sin i$ measurements to those from Reiners \& Basri (2008) for objects with spectral types later than or equal to L4 with a two-sided K-S test indicates a probability of $31 \%$ that the two samples are drawn from the same underlying distribution.

Studying the kinematics of a population of stars, in particular their three-dimensional motions, is a powerful tool for understanding the age of that population as well as for identifying subgroups that may have distinct histories from the main population. As a result of gravitational interactions, the velocity dispersion of a population of stars is expected to increase with time as it orbits in the galaxy. Models of UCDs, and brown dwarfs in particular, have an inherent mass-age degeneracy that makes it difficult to differentiate between a old low-mass star and young brown dwarfs. This means that very few individual

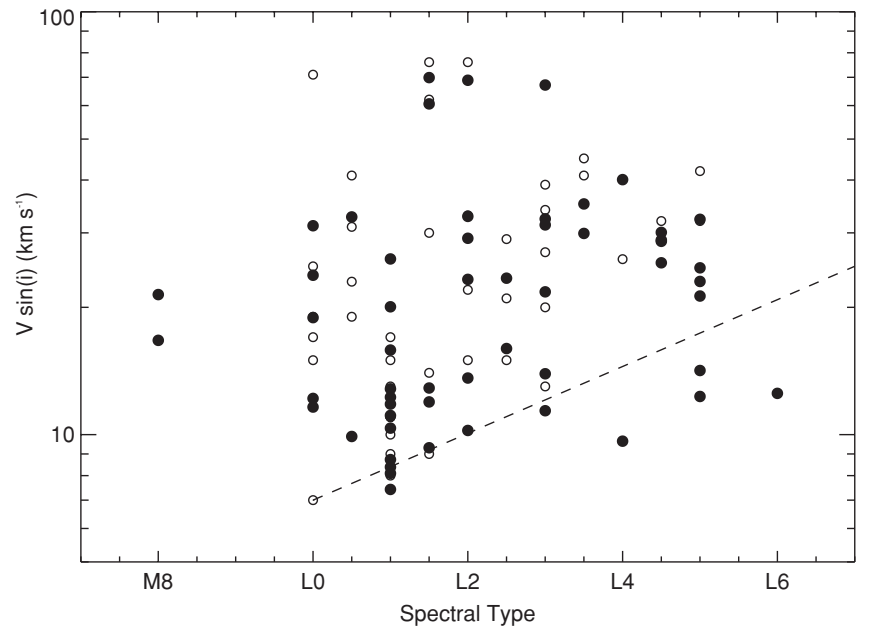

Figure 16. Optical spectral type compared to our measured $V \sin i$ (solid points) along with those from Reiners \& Basri (2008) (open circles). The dashed line corresponds to the $V \sin i$ lower envelope suggested by Reiners \& Basri (2008). We found four examples slowly rotating $\left(V \sin i<15 \mathrm{~km} \mathrm{~s}^{-1}\right)$ objects with mid-L spectral types.

UCDs have reliable age estimates. Since UCDs are intrinsically very faint, the relatively bright UCDs we observe are necessarily close to the Sun and most have reliable proper motion, and sometimes parallax, measurements. Using these measurements, the statistical properties of the kinematics of the UCD population can be determined and the age of the population estimated using calibrated age-dispersion relations (Wielen 1977). Faherty et al. (2009) present a analysis of the space velocities of 841 UCDs. In this work the authors do not have RV information but rely on photometric distance estimates combined with proper motions to estimate the tangential velocity, $V_{\tan }$. As pointed out by Reiners \& Basri (2009), the analysis by Faherty et al. (2009) applies the results from Wielen (1977) by assuming $\sigma_{\text {tot }}=\sqrt{\left\langle V_{\text {tot }}^{2}\right\rangle-\left\langle V_{\text {tot }}\right\rangle^{2}}$ when in reality the dispersion of the total velocity must be determined from the dispersions of the individual velocity components.

We combined our RVs with proper motion and parallax measurements from the literature and photometric distance estimates from Faherty et al. (2009) to estimate the total space velocity, $V_{\text {tot }}$, as well as $U, V, W$ components following the transformation presented by Johnson \& Soderblom (1987). We followed the right handed convention (positive $U$ is toward the galactic center) and we did not apply a correction to the local standard of rest. The distribution of $V_{\text {tot }}=\left(U^{2}+V^{2}+W^{2}\right)^{1 / 2}$ for 55 of our targets with distance and proper motion estimates from the literature is shown in Figure 18 and the velocity components are listed in Table 10. The distribution of $V_{\text {tot }}$ is approximately Gaussian with a significant tail of targets with $V_{\text {tot }}>90 \mathrm{~km} \mathrm{~s}^{-1}$. Excluding these high-velocity UCDs, we found that the average space velocity of the UCDs in our sample is $\left\langle V_{\text {tot }}\right\rangle=40.34 \pm 0.72 \mathrm{~km} \mathrm{~s}^{-1}$, similar to the mean L dwarf space velocity found by Zapatero Osorio et al. (2007). We estimated the velocity dispersions of our sample in each velocity component using a bootstrap simulation taking into account the reported errors on all of the measured quantities used to calculate $U, V$, and $W$. We calculated the $|W|$-weighted dispersions (Equations (1)-(3) in Wielen 1977) and estimated $\sigma_{\text {tot }}=\left(\sigma_{U}^{2}+\sigma_{V}^{2}+\sigma_{W}^{2}\right)^{1 / 2}$ excluding the high-velocity UCDs. Based on the results of the bootstrap simulation, we estimated $\sigma_{\text {tot }}=52.3 \pm 1.7 \mathrm{~km} \mathrm{~s}^{-1}$. We used Equation (16) from Wielen 


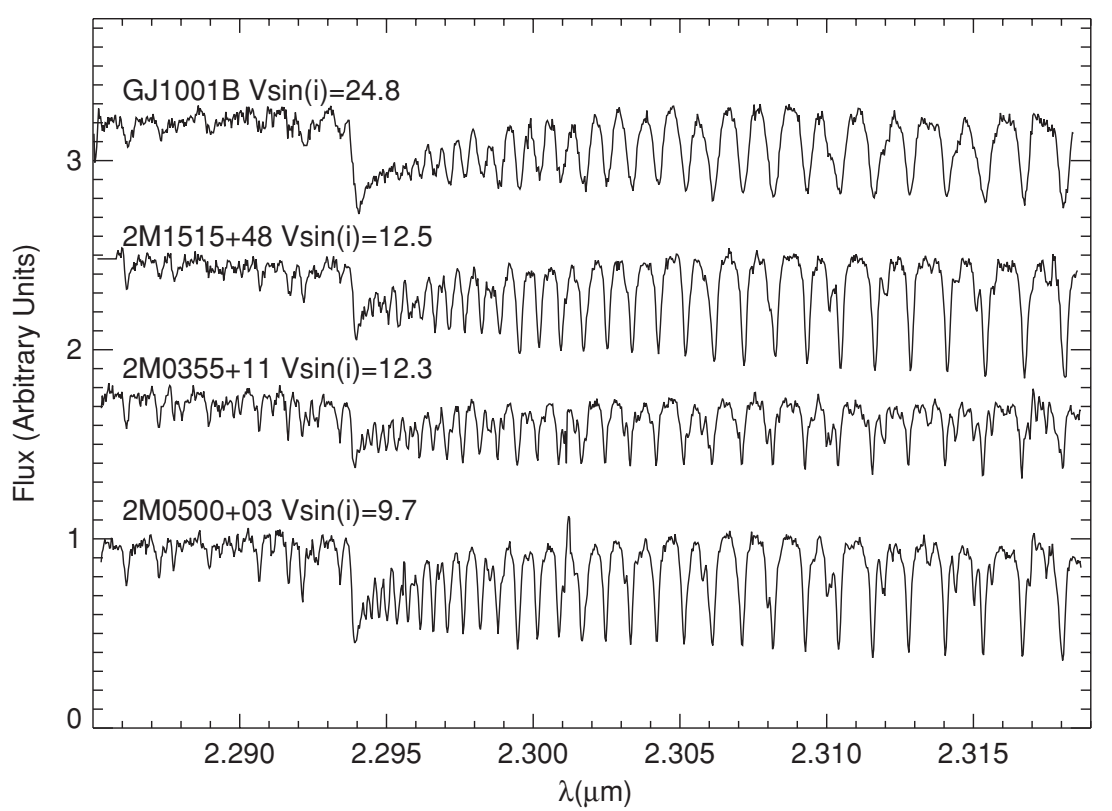

Figure 17. NIRSPEC spectra of three slowly rotating mid-L dwarfs after removal of the telluric features. For comparison, the L5 dwarf GJ $1001 \mathrm{~B}$ with $V \sin i=24.8 \pm 0.4 \mathrm{~km} \mathrm{~s}^{-1}$ is also shown. 2M1515+48 (L6), 2M0355+11 (L5), and 2M0500+03(L4) all appear to have projected rotation velocities smaller than that of GJ 1001B.

(1977; Equation (3) from Reiners \& Basri 2009) to estimate the age of our sample to be $5.0_{-0.6}^{+0.7} \mathrm{Gyr}$ (95\% confidence) not including any systematic uncertainty in the age-dispersion relation. This age is somewhat larger than the $3.1 \mathrm{Gyr}$ estimated by Reiners \& Basri (2009) for their sample of M7-M9.5 dwarfs, though they conclude that $10 \%$ of their objects are actually young brown dwarfs. Our sample of mainly L dwarfs is lower in mass and may have less contamination from young objects, possibly contributing to the differences in the estimated ages of our two samples. Our results are in excellent agreement with those of Seifahrt et al. (2010) who used a similar method to estimate the age for a sample of $43 \mathrm{~L}$ dwarfs. They also estimate the kinematic age of their sample to be approximately $5 \mathrm{Gyr}$ and discuss the implications of this result. Since nearby $M$ dwarfs appear to have an age of approximately 3 Gyr it is surprising that early L dwarfs, which, if below the hydrogen burning mass limit, will dim substantially and move to later spectral types as they age, should be found to be older than $\mathrm{M}$ dwarfs. Possibly explanations for this proposed by Seifahrt et al. (2010) include different initial velocity dispersions for $\mathrm{M}$ and $\mathrm{L}$ dwarfs, the influence of kinematic outliers in the estimation of the velocity dispersions, and non-Gaussianity in the velocity distributions.

The high-velocity UCDs that we excluded from the $V_{\text {tot }}$ analysis are themselves potentially very interesting. Objects with high velocities may be subdwarfs from an older, lowermetallicity thick disk or halo population. In addition to LSR1610 we identified eight $\mathrm{L}$ dwarfs with $V_{\text {tot }}>90 \mathrm{~km} \mathrm{~s}^{-1}$ listed in Table 11. LSR1610 was one of the first known low-mass subdwarfs, and its $V_{\text {tot }}=243.3 \pm 2.0 \mathrm{~km} \mathrm{~s}^{-1}$ exceeds all of the rest of the objects in our sample and based on its velocity components it is considered to be a member of the halo population. In Figure 19, we compare the estimated UVW velocities to velocity ellipsoids for the thin disk and thick disk populations. While the majority of the UCDs in our sample have kinematics consistent with the local thin disk population, seven have large negative $V$ velocities, falling outside the $2 \sigma$ velocity ellipsoid for the thin disk $\left(V<-55 \mathrm{~km} \mathrm{~s}^{-1}\right)$,

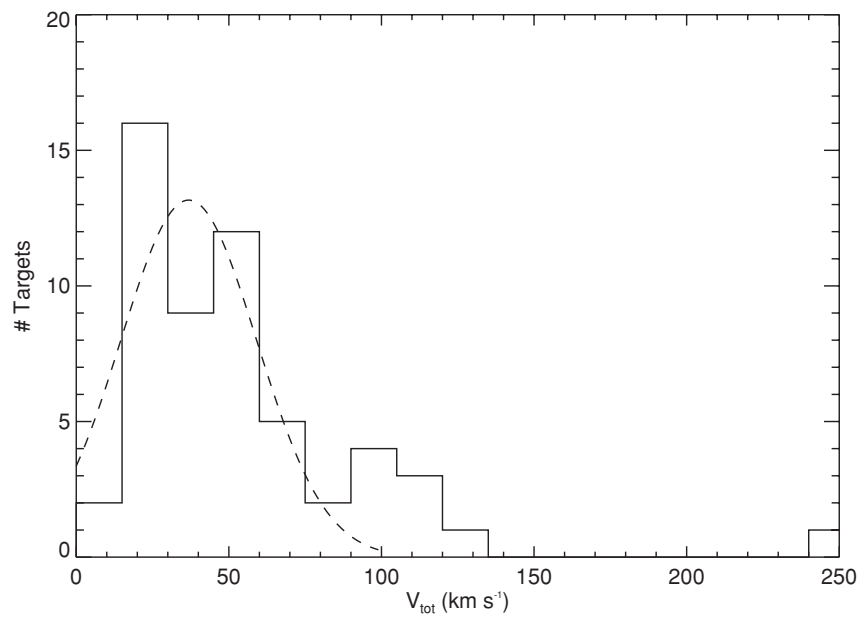

Figure 18. Distribution of three-dimensional space velocities $\left(V_{\text {tot }}\right)$ of the objects in our sample along with a best-fit Gaussian distribution. Excluding the population of high-velocity L dwarfs with $V_{\text {tot }}>90 \mathrm{~km} \mathrm{~s}^{-1}$, we estimate the width of the $|W|$-weighted $V_{\text {tot }}$ distribution to be $\sigma_{V_{\text {tot }}}=52.3 \pm 1.7 \mathrm{~km} \mathrm{~s}^{-1}$. The high-velocity UCD LSR $1610-0040$ is shown near $250 \mathrm{~km} \mathrm{~s}^{-1}$. We note that $\sigma_{\text {tot }}=\left(\sigma_{U}^{2}+\sigma_{V}^{2}+\sigma_{W}^{2}\right)^{1 / 2} \neq \sqrt{\left\langle V_{\text {tot }}^{2}\right\rangle-\left\langle V_{\text {tot }}\right\rangle^{2}}$.

making them candidates for membership in the older thick disk population.

\section{TIGHT BINARY RATE}

Thanks mainly to direct imaging surveys, we now know that the population of UCD binaries has several significant differences from that of binaries composed of Sun-like stars. These differences are summarized in Allen (2007) and Burgasser et al. (2007): (1) UCD binaries tend to have mass ratios $q \sim 1 \mathrm{com}-$ pared to $q \sim 0.3$ for Sun-like stars. (2) There are very few UCD binaries with $a>15-20$ AU. (3) For UCDs the distribution of binary separation peaks around $a \sim 7$ AU compared to $a \sim 30$ AU for Sun-like stars. (4) The overall fraction of binaries is $\sim 20 \%$ for UCDs compared to $60 \%$ for Sun-like stars. 
Table 10

UVW Velocity Components

\begin{tabular}{|c|c|c|c|c|}
\hline ID & $\begin{array}{c}U \\
\left(\mathrm{~km} \mathrm{~s}^{-1}\right) \\
\end{array}$ & $\begin{array}{c}V \\
\left(\mathrm{~km} \mathrm{~s}^{-1}\right) \\
\end{array}$ & $\begin{array}{c}W \\
\left(\mathrm{~km} \mathrm{~s}^{-1}\right) \\
\end{array}$ & $\begin{array}{c}V_{\text {tot }} \\
\left(\mathrm{km} \mathrm{s}^{-1}\right)\end{array}$ \\
\hline GJ1001B & $1.37 \pm 0.42$ & $-109.16 \pm 5.55$ & $-20.03 \pm 0.62$ & $110.99 \pm 5.61$ \\
\hline $2 \mathrm{M} 0015+35$ & $13.03 \pm 2.02$ & $-42.88 \pm 1.76$ & $-6.53 \pm 2.54$ & $45.29 \pm 3.69$ \\
\hline $2 \mathrm{M} 0036+18$ & $-42.15 \pm 0.41$ & $-5.80 \pm 0.22$ & $-11.59 \pm 0.09$ & $44.10 \pm 0.47$ \\
\hline $2 \mathrm{M} 0045+16$ & $-22.96 \pm 1.79$ & $-13.41 \pm 1.30$ & $-3.98 \pm 0.59$ & $26.88 \pm 2.29$ \\
\hline $2 \mathrm{M} 0141+18$ & $-48.92 \pm 3.08$ & $-21.11 \pm 2.97$ & $-10.94 \pm 1.08$ & $54.39 \pm 4.41$ \\
\hline 2M0144-07 & $-20.82 \pm 2.62$ & $-46.41 \pm 4.43$ & $3.88 \pm 0.84$ & $51.01 \pm 5.22$ \\
\hline $2 \mathrm{M} 0213+44$ & $19.54 \pm 5.37$ & $-17.52 \pm 5.40$ & $-8.81 \pm 5.69$ & $27.68 \pm 9.51$ \\
\hline $2 \mathrm{M} 0227-16$ & $-36.24 \pm 1.55$ & $-61.75 \pm 3.01$ & $-31.17 \pm 1.21$ & $78.09 \pm 3.60$ \\
\hline $2 \mathrm{M} 0228+25$ & $-39.56 \pm 2.87$ & $-14.08 \pm 3.28$ & $-0.38 \pm 2.15$ & $41.99 \pm 4.86$ \\
\hline $2 \mathrm{M} 0235-23$ & $-12.45 \pm 0.20$ & $-8.45 \pm 0.16$ & $-10.38 \pm 0.16$ & $18.28 \pm 0.30$ \\
\hline $2 \mathrm{M} 0251-03$ & $15.68 \pm 1.58$ & $-120.09 \pm 10.09$ & $-14.14 \pm 1.26$ & $121.93 \pm 10.29$ \\
\hline 2M0320-04 & $52.03 \pm 10.22$ & $-26.46 \pm 5.39$ & $-44.23 \pm 8.68$ & $73.24 \pm 14.45$ \\
\hline $2 \mathrm{M} 0352+02$ & $-55.97 \pm 2.22$ & $7.41 \pm 2.53$ & $12.77 \pm 2.73$ & $57.89 \pm 4.34$ \\
\hline $2 \mathrm{M} 0355+11$ & $-6.29 \pm 1.06$ & $-22.19 \pm 2.91$ & $-14.79 \pm 1.32$ & $27.40 \pm 3.37$ \\
\hline $2 \mathrm{M} 0500+03$ & $-4.82 \pm 1.34$ & $-20.41 \pm 1.79$ & $-16.43 \pm 1.48$ & $26.64 \pm 2.68$ \\
\hline $2 \mathrm{M} 0523-14$ & $-16.27 \pm 0.93$ & $-3.26 \pm 0.95$ & $3.30 \pm 1.17$ & $16.92 \pm 1.77$ \\
\hline 2M0539-00 & $-21.86 \pm 0.37$ & $3.31 \pm 0.32$ & $13.84 \pm 0.55$ & $26.09 \pm 0.74$ \\
\hline LSR0602+39 & $-11.90 \pm 0.38$ & $-26.51 \pm 2.59$ & $-2.47 \pm 0.74$ & $29.16 \pm 2.72$ \\
\hline $2 \mathrm{M} 0652+47$ & $7.38 \pm 0.93$ & $5.97 \pm 2.65$ & $-4.08 \pm 2.16$ & $10.34 \pm 3.55$ \\
\hline $2 \mathrm{M} 0700+31$ & $42.13 \pm 0.15$ & $-28.97 \pm 0.95$ & $-15.62 \pm 0.25$ & $53.46 \pm 1.00$ \\
\hline $2 \mathrm{M} 0717+57$ & $13.88 \pm 1.17$ & $-2.03 \pm 1.34$ & $-8.07 \pm 2.77$ & $16.19 \pm 3.29$ \\
\hline $2 \mathrm{M} 0746+20$ & $-55.63 \pm 0.14$ & $-15.04 \pm 0.07$ & $-1.74 \pm 0.09$ & $57.65 \pm 0.19$ \\
\hline $2 \mathrm{M} 0828-13$ & $-38.71 \pm 3.50$ & $-13.49 \pm 1.10$ & $-20.68 \pm 3.91$ & $45.91 \pm 5.36$ \\
\hline $2 \mathrm{M} 0835-08$ & $-33.22 \pm 2.48$ & $-14.77 \pm 1.33$ & $0.89 \pm 1.65$ & $36.37 \pm 3.27$ \\
\hline $2 \mathrm{M} 0847-15$ & $15.84 \pm 1.28$ & $-10.68 \pm 0.74$ & $1.79 \pm 1.01$ & $19.19 \pm 1.79$ \\
\hline 2M0921-21 & $19.43 \pm 3.85$ & $-94.59 \pm 2.04$ & $5.77 \pm 2.12$ & $96.74 \pm 4.84$ \\
\hline $2 \mathrm{M} 0953-10$ & $-4.72 \pm 1.83$ & $-21.15 \pm 1.47$ & $2.14 \pm 1.80$ & $21.77 \pm 2.96$ \\
\hline $2 \mathrm{M} 1022+58$ & $-65.37 \pm 14.65$ & $60.85 \pm 9.29$ & $-46.79 \pm 10.47$ & $100.82 \pm 20.26$ \\
\hline 2M1045-01 & $-35.71 \pm 2.41$ & $-14.22 \pm 1.00$ & $-12.83 \pm 1.33$ & $40.52 \pm 2.93$ \\
\hline $2 \mathrm{M} 1048+01$ & $-24.91 \pm 1.57$ & $-34.50 \pm 1.47$ & $-1.43 \pm 1.47$ & $42.58 \pm 2.61$ \\
\hline $2 \mathrm{M} 1051+56$ & $-15.81 \pm 4.00$ & $-31.47 \pm 2.39$ & $-16.95 \pm 1.99$ & $39.09 \pm 5.06$ \\
\hline $2 \mathrm{M} 1108+68$ & $-41.59 \pm 8.42$ & $-36.59 \pm 4.47$ & $-17.93 \pm 4.02$ & $58.23 \pm 10.34$ \\
\hline $2 \mathrm{M} 1112+35$ & $-20.75 \pm 0.45$ & $-25.17 \pm 0.50$ & $-13.83 \pm 0.31$ & $35.43 \pm 0.74$ \\
\hline $2 \mathrm{M} 1155-37$ & $35.42 \pm 2.09$ & $-50.55 \pm 1.19$ & $-22.97 \pm 3.41$ & $65.86 \pm 4.17$ \\
\hline $2 \mathrm{M} 1203+00$ & $-83.82 \pm 8.69$ & $-67.49 \pm 7.03$ & $-29.92 \pm 3.11$ & $111.70 \pm 11.60$ \\
\hline $2 \mathrm{M} 1221+02$ & $51.86 \pm 9.23$ & $-23.29 \pm 5.19$ & $-27.25 \pm 3.49$ & $63.04 \pm 11.15$ \\
\hline $2 \mathrm{M} 1300+19$ & $-3.87 \pm 1.22$ & $-98.51 \pm 7.00$ & $-26.40 \pm 0.67$ & $102.06 \pm 7.14$ \\
\hline $2 \mathrm{M} 1305-25$ & $-20.27 \pm 0.86$ & $-19.39 \pm 0.67$ & $6.24 \pm 0.33$ & $28.74 \pm 1.14$ \\
\hline $2 \mathrm{M} 1425-36$ & $-5.05 \pm 1.09$ & $-22.47 \pm 2.23$ & $-10.26 \pm 1.50$ & $25.22 \pm 2.90$ \\
\hline $2 \mathrm{M} 1439+19$ & $-85.11 \pm 0.53$ & $-42.92 \pm 0.28$ & $17.75 \pm 0.31$ & $96.96 \pm 0.68$ \\
\hline $2 \mathrm{M} 1506+13$ & $-41.64 \pm 3.22$ & $-48.84 \pm 3.82$ & $36.85 \pm 2.92$ & $74.01 \pm 5.79$ \\
\hline 2M1507-16 & $-26.41 \pm 0.14$ & $-17.10 \pm 0.12$ & $-39.67 \pm 0.12$ & $50.63 \pm 0.22$ \\
\hline $2 \mathrm{M} 1515+48$ & $-107.23 \pm 9.50$ & $-30.47 \pm 1.63$ & $-2.76 \pm 2.11$ & $111.51 \pm 9.86$ \\
\hline 2M1539-05 & $37.58 \pm 1.20$ & $35.00 \pm 2.43$ & $-4.76 \pm 1.62$ & $51.58 \pm 3.16$ \\
\hline $2 \mathrm{M} 1552+29$ & $-11.09 \pm 1.80$ & $-22.92 \pm 1.96$ & $-3.75 \pm 1.54$ & $25.74 \pm 3.08$ \\
\hline $2 \mathrm{M} 1555-09$ & $52.11 \pm 3.08$ & $3.19 \pm 1.25$ & $-57.00 \pm 5.03$ & $77.29 \pm 6.03$ \\
\hline LSR1610-0040 & $-32.11 \pm 0.82$ & $-232.9 \pm 1.7$ & $-62.75 \pm 0.51$ & $243.3 \pm 2.0$ \\
\hline $2 \mathrm{M} 1645-13$ & $33.00 \pm 0.81$ & $-46.83 \pm 4.46$ & $-0.08 \pm 1.36$ & $57.28 \pm 4.74$ \\
\hline $2 \mathrm{M} 1658+70$ & $24.56 \pm 0.23$ & $-42.29 \pm 0.29$ & $22.43 \pm 0.45$ & $53.81 \pm 0.59$ \\
\hline $2 \mathrm{M} 1705-05$ & $14.25 \pm 0.53$ & $2.95 \pm 0.88$ & $-3.80 \pm 0.98$ & $15.05 \pm 1.42$ \\
\hline $2 \mathrm{M} 1731+27$ & $-5.28 \pm 1.35$ & $-29.71 \pm 1.25$ & $-13.28 \pm 1.14$ & $32.97 \pm 2.16$ \\
\hline $2 \mathrm{M} 1807+50$ & $7.46 \pm 1.41$ & $-0.67 \pm 1.12$ & $-5.41 \pm 1.88$ & $9.24 \pm 2.61$ \\
\hline $2 \mathrm{M} 2036+10$ & $24.26 \pm 2.30$ & $4.73 \pm 1.75$ & $-5.27 \pm 1.68$ & $25.27 \pm 3.34$ \\
\hline $2 \mathrm{M} 2104-10$ & $-38.79 \pm 3.43$ & $-29.37 \pm 2.65$ & $-33.36 \pm 5.82$ & $58.99 \pm 7.26$ \\
\hline $2 \mathrm{M} 2224-01$ & $-9.62 \pm 0.11$ & $-63.97 \pm 0.39$ & $-6.01 \pm 0.34$ & $64.97 \pm 0.52$ \\
\hline
\end{tabular}

Notes. UVW velocity components and $V_{\text {tot }}$ for the portion of our sample with distance estimates and proper motions in Faherty et al. (2009). We use a right handed system with $U>0$ toward the galactic center. Errors are derived from a Monte Carlo simulation that includes the errors on RV, distance, and proper motion.

Using a Bayesian approach that assumes a parameterized underlying distribution for the properties of UCD binary systems, Allen (2007) estimated that there is likely a small but signifi- cant population of small separation ( $a<1 \mathrm{AU}) \mathrm{UCD}$ binaries that are not detected by imaging surveys or the RV surveys that have been conducted to date. Systematic RV surveys like our 


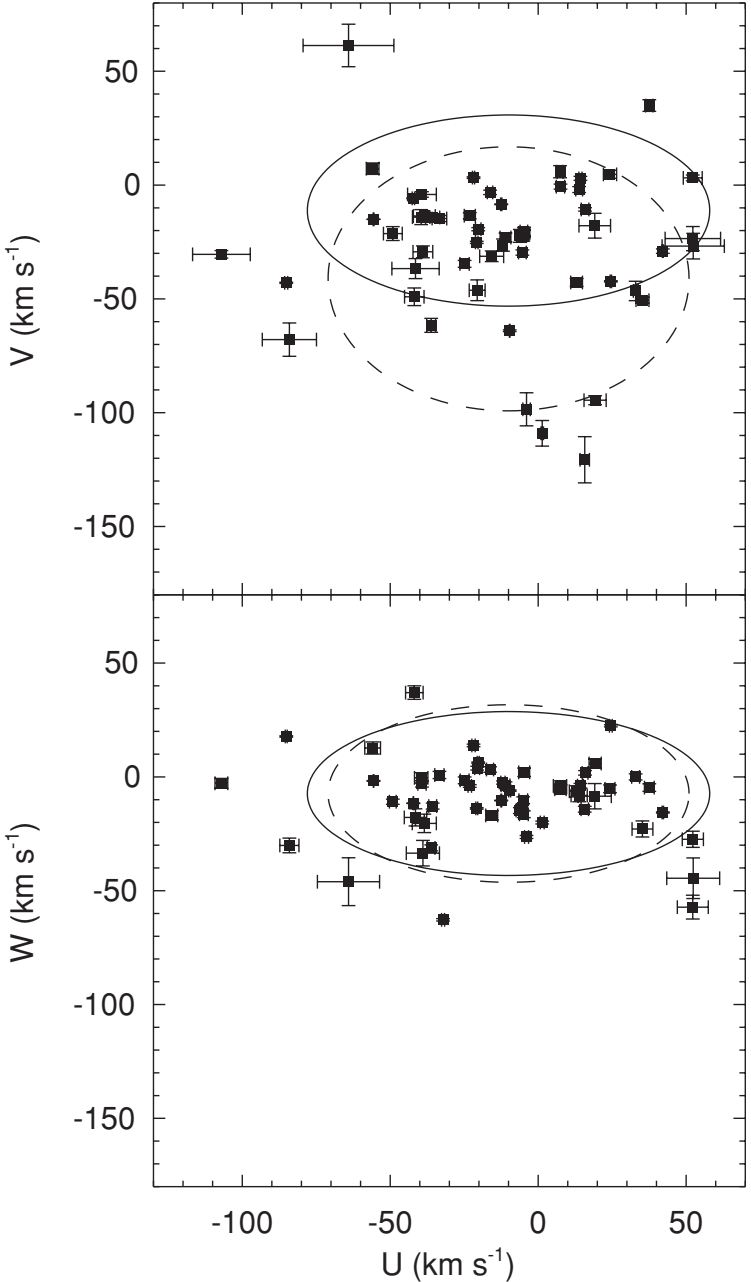

Figure 19. Velocities of our targets in the $U-V$ and $U-W$ planes. The solid line is the $2 \sigma$ velocity ellipsoid for the thin disk population and the dashed line is the $1 \sigma$ ellipsoid for the thick disk population (Binney \& Merrifield 1998). Large negative $V$ velocities are a possible indication of thick disk membership.

NIRSPEC survey are important for constraining the binary fraction in this part of parameter space. An equal-mass UCD binary $\left(M_{1}=M_{2}=0.08 M_{\odot}\right)$ with an average separation of $a=1 \mathrm{AU}$ has an orbital period of seven years and an RV semi-amplitude $K_{1}=K_{2}=1.6 \mathrm{~km} \mathrm{~s}^{-1}$, potentially detectable in our survey. We carried out a Monte Carlo simulation to estimate how efficiently our survey detects tight binaries and the overall rate of such systems.

For our simulation, we used a sub-sample of 40 targets from our survey meeting the following criteria: $K<12.5$, not a known binary, not a known subdwarf, observations on two or more epochs, and total time span of observations more then 350 days. Our simulation is similar in nature to the simulations of Maxted \& Jeffries (2005), though we made some simplifying assumptions. Using the actual times of observations of these 40 targets, as well as the actual RV error estimates for each observation, we generated $10^{4}$ hypothetical surveys where each of the 40 targets is a tight binary. For the mass ratio, $q=M_{2} / M_{1}$, we used a power-law distribution with exponent $\gamma=1.8$ (Allen 2007). We assumed that each of our UCDs has a mass of $0.08 M_{\odot}$ and used a uniform distribution in $a$ between 0.01 and 1.0 AU to calculate spectroscopic binary orbits with random phase, argument of periapsis, $\omega$, and inclination. We assumed a distribution of eccentricity that is uniform between $e=0.0$ and
Table 11

High-velocity UCDs

\begin{tabular}{lccc}
\hline \hline \multicolumn{1}{c}{ Name } & $\begin{array}{c}V_{\text {tot }} \\
\left(\mathrm{km} \mathrm{s}^{-1}\right)\end{array}$ & $\begin{array}{c}V \\
\left(\mathrm{~km} \mathrm{~s}^{-1}\right)\end{array}$ & $\begin{array}{c}V \sin i \\
\left(\mathrm{~km} \mathrm{~s}^{-1}\right)\end{array}$ \\
\hline GJ1001B & $110.99 \pm 5.61$ & $-109.16 \pm 5.55$ & $24.80 \pm 0.40$ \\
2M0227-16 & $78.09 \pm 3.60$ & $-61.75 \pm 3.01$ & $<9$ \\
2M0251-03 & $121.93 \pm 10.29$ & $-120.09 \pm 10.09$ & $21.76 \pm 0.38$ \\
2M0921-21 & $96.74 \pm 4.84$ & $-94.59 \pm 2.04$ & $11.95 \pm 0.49$ \\
2M1022+58 & $100.82 \pm 20.26$ & $60.85 \pm 9.29$ & $11.81 \pm 0.21$ \\
2M1203+00 & $111.70 \pm 11.60$ & $-67.49 \pm 7.03$ & $31.33 \pm 0.55$ \\
2M1300+19 & $102.06 \pm 7.14$ & $-98.51 \pm 7.00$ & $12.83 \pm 2.10$ \\
2M1439+19 & $96.96 \pm 0.68$ & $-42.92 \pm 0.28$ & $11.10 \pm 0.24$ \\
2M1515+48 & $111.1 \pm 9.86$ & $-30.47 \pm 1.63$ & $12.52 \pm 1.65$ \\
LSR1610-0040 & $243.30 \pm 2.00$ & $-232.96 \pm 1.70$ & $16.84 \pm 3.11$ \\
2M2224-01 & $64.97 \pm 0.52$ & $-63.97 \pm 0.39$ & $25.49 \pm 0.41$ \\
\hline
\end{tabular}

Notes. High-velocity ( $V_{\text {tot }}>90 \mathrm{~km} \mathrm{~s}^{-1}$ or $V<-55 \mathrm{~km} \mathrm{~s}^{-1}$ ) UCDs identified in our NIRSPEC survey. These objects have space velocities exceeding the velocities of the local stellar population, indicating that they may be part of a distinct, older thick disk population. One of the high-velocity targets, LSR1610-0040, is an L subdwarf.

$e=0.6$. We generated synthetic data based on the binary orbits, the actual times of the NIRSPEC observations, and adding noise based on the scaled error estimates at each epoch, $\sigma_{\mathrm{RV}}$. Since we have already adjusted our empirical error estimates so that they are a good description of the overall statistical properties of our data, we feel that a synthetic data set generated in this way is a fair representation of a potential survey. We excluded epochs after 2009 April since these observations were primarily for follow-up of suspected binary systems. To estimate the efficiency with which such systems would have been detected in our survey, we counted the number of binary systems detected in each simulated survey with a conservative requirement that $P\left(\chi_{\mathrm{RV}}^{2} \leqslant\right)>0.999$ to qualify as a detection. Based on these simulations, we estimated that our overall average efficiency in detecting systems with $0.01 \mathrm{AU}<a<1.0 \mathrm{AU}$ is $94 \%$. We found that this efficiency is relatively insensitive to the assumed mass of the primary and, since it is peaked near 1.0, the assumed mass ratio distribution.

Our survey contains 43 targets that would have been included in the sample based on the above criteria alone and assuming that none of these systems passed the magnitude cut because they are unknown binaries. Within this sample, we found one definite tight SB1 system (2M0320-04) and one possible SB1 system (2M1507-16), though if this system is a genuine SB1 then the separation may be larger than $a=1 \mathrm{AU}$. The system $2 \mathrm{M} 0746+20$ was a known astrometric binary, with $a>1 \mathrm{AU}$, and LSR1610 -0040 and LSR0602+39 were known subdwarfs. Based on the estimated detection efficiency, a flat prior on the binary fraction, and the detection of one (possibly two) tight binaries, we use the binomial distribution to make a Bayesian estimate of the fraction of late-M and $\mathrm{L}$ dwarf systems in tight binaries (90\% confidence) of $2.5_{-1.6}^{+8.6} \%\left(5.0_{-3.0}^{+9.6} \%\right.$ ). While our sample is small and the number of detected tight binaries is small, our observations are consistent with the overall binary properties from Allen (2007) and in particular with the prediction that $\sim 3 \%-4 \%$ of UCDs are in binaries with $a<1$ AU. Assuming similar underlying statistical distributions to the ones, we used here, Maxted \& Jeffries (2005) combined RV observations from the literature and found a relatively large number of close binaries, requiring an overall binary fraction of $40 \%$, approximately a factor of 2 higher than those from Allen (2007), Joergens (2008), and Basri \& Reiners (2006). While our 


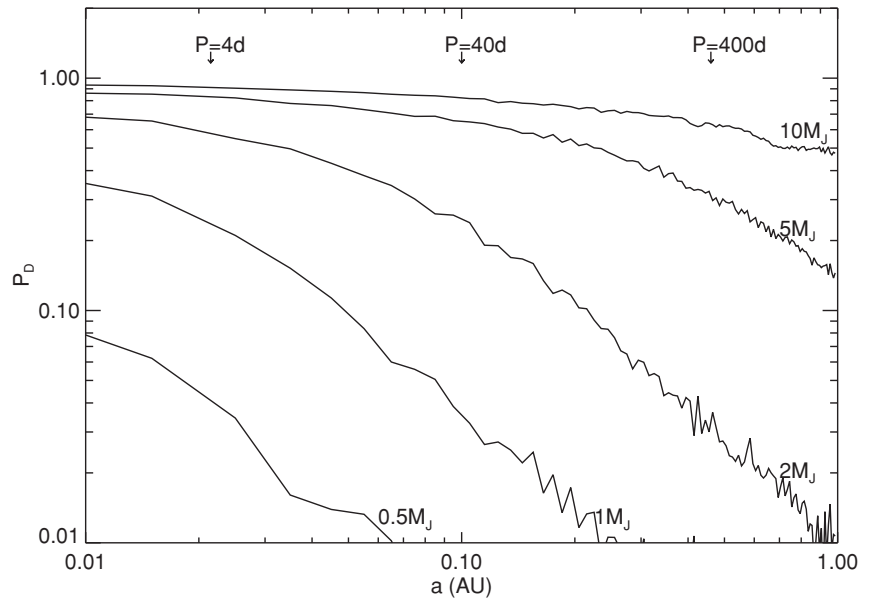

Figure 20. Results of a Monte Carlo simulation designed to estimate the planet detection efficiency of our survey, $P_{D}$. We have sensitivity to Jupiter-mass companions at small orbital separations and the most massive planets out to $a \sim 1.0 \mathrm{AU}$. The Roche limit for these systems is $a>0.002 \mathrm{AU}$ (Blake et al. 2008a).

results are consistent with the lower overall binary fraction, our current constraint does not rule out the higher value.

\subsection{Planet Detection Efficiency}

For at least four reasons, UCDs are excellent targets for planet searches: (1) the semi-amplitude of the Doppler signal, $K_{1}$, increases inversely with the mass of the host $\operatorname{star}\left(K_{1} \propto M_{*}^{-2 / 3}\right)$ for a fixed orbital period and planetary mass. If the level of Doppler precision achieved for F-G-K dwarfs can be achieved for UCDs, then it becomes possible, in principle, to detect super-Earth planets in the so-called Habitable Zone (HZ), where in the absence of an atmosphere the equilibrium temperature of a planet is in the liquid water range. (2) UCDs have low luminosities compared to Sun-like stars, meaning that the HZ moves closer in to the host, serving to boost the Doppler signal of a planet of fixed mass beyond the gain from the decrease in the mass of the primary. (3) Understanding the population of planetary companions to UCDs, and how this population differs from that of planets orbiting F-G-K dwarfs, may allow astronomers to conduct important tests of theories of planet formation and migration. A significant population of giant planets orbiting close to $\mathrm{M}$ and $\mathrm{L}$ dwarfs is not predicted by models of planet formation via core accretion (i.e., Laughlin et al. 2004; Ida \& Lin 2005), though such planets may be formed in the disk instability scenario (Boss 2006). (4) The ratio of the intrinsic brightness of a planet to its host at thermal infrared wavelengths is orders of magnitude larger when that host is a UCD rather than a Sun-like star. The relative proximity to the Sun of the brightest UCDs that we observe also means that planetary companions will have relatively large angular separations on the sky. Favorable contrast ratios and angular separations mean that planetary companions to UCDs will likely be among the best targets for efforts to directly image and study the atmospheres of extrasolar planets at infrared and midinfrared wavelengths using high contrast imaging techniques.

We ran a Monte Carlo simulation to estimate our ability to detect binary systems with small mass ratios $(q<0.2)$ and orbital separations 0.01 AU $<a<1.0 \mathrm{AU}$. While our current RV precision precludes the detection of sub-Jupitermass planets, we found that our survey is sensitive to giant planets and the lowest mass brown dwarfs over a wide range of orbital separations. For this simulation, we assumed a uniform distribution in $a$ from 0.005 to $1.0 \mathrm{AU}$, circular orbits with $e=0$, random inclination, phase, and $\omega$, a primary mass of $M_{1}=0.08 M_{\odot}$, and we tested a range of companion masses $0.5 M_{\mathrm{J}}<M_{2}<15 M_{\mathrm{J}}$. We followed a similar procedure to that described previously in this section to generate $10^{4}$ realization of our survey and inserted spectroscopic orbits with random inclinations into the synthetic data and counted detections using the same $P\left(\chi_{\mathrm{RV}}^{2} \leqslant\right)>0.999$ criteria. The results of this simulation are shown in Figure 20.

With no definitive detections of companions in this mass regime, we are only able to place upper limits on the prevalence of giant planetary companions to UCDs. We found that for small orbital separations, similar to those of the Hot Jupiters found orbiting Sun-like stars (0.01 AU $<a<0.05 \mathrm{AU})$, our survey is very sensitive to giant planets $\left(1 M_{\mathrm{J}}<M_{2}<10 M_{\mathrm{J}}\right)$ and the least massive brown dwarfs. Based on the average detection efficiencies, $P_{D}$, shown in Figure 20, we estimate the following upper limits $(95 \%)$ for the rate of planetary companions, $f_{P}$, at orbital separations $a<0.05 \mathrm{AU}$ : for $1 M_{\mathrm{J}}, f_{p}<0.63$, for $2 M_{\mathrm{J}}, f_{p}<0.17$, for $5 M_{\mathrm{J}}, f_{p}<0.088$, and for $10 M_{\mathrm{J}}$, $f_{p}<0.076$. It is also interesting to consider the case of a companion with mass $15 M_{\mathrm{J}}$, possibly a Y dwarf, for which we find $f_{p}<0.089$ for $a<0.5$ AU. Based on other observational evidence, we would expect the rate of close-in giant companions to be very low. If a system with $q<0.2$ and $a<0.05$ AU can be thought of as a binary system, as opposed to a planetary system, then the mass ratio distribution, separation distribution, and overall binary fraction from Allen (2007) would predict that the frequency of such companions would be exceedingly rare, orbiting fewer than 1 in $10^{6}$ UCDs. There are a number of very massive planets known to orbit at these distances from Sun-like stars, including WASP-19b (Hebb et al. 2010), Corot2b (Bouchy et al. 2008), and WASP-14b (Joshi et al. 2009), though these massive hot Jupiters are relatively rare with a rate of occurrence of $f_{P}<0.005$ (Cumming et al. 2008; Gould et al. 2006a). The analyses of Cumming et al. (2008) and Johnson et al. (2007) both indicate that the overall rate of occurrence of planets decreases with stellar mass, with the rate of planets orbiting $\mathrm{M}$ stars being down by a factor of $\sim 7$ compared to Sun-like stars. While there is no direct observational evidence pertaining to hot Jupiters orbiting UCDs, extrapolating from the observed properties of planets orbiting high-mass stars indicates that the expected rate of occurrence, $f_{p}$, of these companions is probably less than $f_{P}<0.001$.

\section{CONCLUSIONS}

We report the results of an NIR Doppler survey designed to detect unseen companions to UCDs. Infrared RV measurements are a powerful tool for detecting UCD spectroscopic binaries and potentially even giant planets orbiting low-mass stars and brown dwarfs. We have developed a new technique for making $\mathrm{RV}$ measurements at infrared wavelengths using $\mathrm{CH}_{4}$ absorption features in Earth's atmosphere as a simultaneous wavelength reference. We have used this technique to carry out a Doppler survey of UCDs using NIRSPEC on the Keck II telescope. We obtained more than 600 spectra of 59 UCDs on two or more epochs. We forward modeled our spectra using highresolution theoretical UCD templates and the observed telluric spectrum, scaled for airmass, to measure radial velocity, RV, and the projected rotational velocity, $V \sin i$. We estimated our RV precision to be $50 \mathrm{~m} \mathrm{~s}^{-1}$ for bright, slowly rotating 
M dwarfs and 100-300 $\mathrm{m} \mathrm{s}^{-1}$ for slowly rotating UCDs. Within the framework of the photon-limited Doppler precision, we investigated different sources of noise that may limit our RV precision. Neglecting secular changes in $\mathrm{CH}_{4}$ absorption features due to winds or changes is barometric pressure, we estimated that for our typical spectra $(\mathrm{S} / \mathrm{N} \sim 75)$, the telluric features themselves limit RV precision to $\sigma_{\mathrm{RV}}>60 \mathrm{~m} \mathrm{~s}^{-1}$. We estimated that the overall precision of our RV measurements $\left(\sigma_{\mathrm{RV}}\right)$ is within a factor of 2 of the photon limit. There are several potential sources for this excess noise including changes in the symmetry of the NIRSPEC LSF, changes in the $\mathrm{CH}_{4}$ absorption features themselves, episodes of high read noise in the detector, mismatch between the theoretical templates and the actual UCD spectra, and changes in the phase and amplitude of the "fringing" pattern seen in our NIRSPEC spectra. We explored avenues for mitigating these instrumental sources of RV variations, but ultimately were unable to improve the overall RV precision to the photon limit.

We identified one new spectroscopic binary, one candidate binary, and obtained RV measurements of two astrometric binaries from the literature. The candidate spectroscopic binary, 2M1507-16, shows a long-term RV trend and the absence of any observed companion in high-resolution imaging observations may indicate that the system is a low-amplitude spectroscopic binary with a period $P>2500$ days. Due to the proximity of the primary to the Sun, this object is an excellent target for ongoing efforts to directly image small mass ratio UCD binary systems.

We also investigated the rotation and kinematics of the UCDs in our sample. Our estimates of $V \sin i$ are consistent with those in the literature. Unlike Reiners \& Basri (2008), our sample contains at least four mid-L dwarfs that are rotating at $V \sin i<15 \mathrm{~km} \mathrm{~s}^{-1}$, though we conclude that our two samples are statistically consistent. With proper motions and distance estimates from the literature, we were able to use our RV measurements to calculate the full three-dimensional space velocities of $55 \mathrm{~L}$ dwarfs and estimate the width of the distribution of the measured values of $V_{\text {tot }}$. The dispersion of this velocity distribution is expected to be directly related to the age of a stellar population. We estimated the kinematic age of our sample to be $5.0_{-0.6}^{+0.7}$ Gyr. Based on simulations of our detection efficiency we estimated the rate of tight binaries $(a<1 \mathrm{AU})$ to be $2.5_{-1.6}^{+8.6} \%$, consistent with an overall binary fraction of $20 \%$ and a predicted tight binary fraction of $3 \%-4 \%$ given by Allen (2007). Finally, we simulated our ability to detect giant planetary companions in short-period orbits and concluded that a large population of giant planets $\left(1 M_{\mathrm{J}}<M_{2}<10 M_{\mathrm{J}}\right)$ in close $(a<0.05 \mathrm{AU})$ orbits would have been detected in our survey. Specifically, we find that the rate of occurrence of companions with $M_{2}>1 M_{\mathrm{J}}$ and $a<0.05 \mathrm{AU}$ is less than $62 \%$ and that the rate of occurrence of more massive companions with $M_{2}>15 M_{\mathrm{J}}$ and $a<0.5 \mathrm{AU}$ is less than $8.8 \%$

We thank D. Saumon and M. Marley for providing the library of high-resolution synthetic UCD spectra that made this work possible as well as for thoughtful comments on this manuscript. We are grateful to the referee, A. Reiners, for his careful reading of our manuscript and his many helpful suggestions for improvements. We thank D. Spiegel for several helpful discussions about statistics. It is a pleasure to acknowledge D. Latham, C. Stubbs, G. Torres for their guidance throughout this work. We also thank D. Finkbeiner, A. Loeb, and K. Stassun for helpful discussions and suggestions for improvements to this work. We also thank J. Bailey for helpful discussions throughout the process of reducing and analyzing the NIRSPEC data. C.H.B. acknowledges financial support from the Harvard Origins of Life Initiative, NExSci, and the NSF Astronomy \& Astrophysics Postdoctoral Fellowship program. Data presented herein were obtained at the W. M. Keck Observatory in part with telescope time allocated to the National Aeronautics and Space Administration through the agency(tm)s scientific partnership with the California Institute of Technology and the University of California. The Observatory was made possible by the generous financial support of the W. M. Keck Foundation. We thank G. Hill, J. Lyke, and the Keck staff for their support over the course of this program. The Keck Observatory was made possible by the generous financial support of the W.M. Keck Foundation. The authors recognize and acknowledge the very significant cultural role and reverence that the summit of Mauna Kea has always had within the indigenous Hawaiian community. We are most fortunate to have the opportunity to conduct observations from this mountain. This research has benefited from the M, $\mathrm{L}$, and $\mathrm{T}$ dwarf compendium housed at DwarfArchives.org and maintained by C. Gelino, D. Kirkpatrick, and A. Burgasser.

Facilities: Keck:II

\section{REFERENCES}

Ackerman, A. S., \& Marley, M. S. 2001, ApJ, 556, 872

Allen, P. 2007, ApJ, 668, 492

Apai, D., Pascucci, I., Bouwman, J., Natta, A., Henning, T., \& Dullemond, C. P. 2005, Science, 310,834

Bailer-Jones, C. A. L. 2004, A\&A, 419, 703

Bailey, J., Butler, R., Tinney, C. G., Jones, H. R. A., O’Toole, S., Carter, B. D., \& Marcy, G. W. 2009, ApJ, 690, 743

Balthasar, H., Thiele, U., \& Woehl, H. 1982, A\&A, 114, 357

Baraffe, I., Chabrier, G., Allard, F., \& Hauschildt, P. H. 1998, A\&A, 337, 403

Baraffe, I., Chabrier, G., Barman, T. S., Allard, F., \& Hauschildt, P. H. 2003, A\&A, 402, 701

Basri, G., Mohanty, S., Allard, F., Hauschildt, P. H., Delfosse, X., Martín, E. L., Forveille, T., \& Goldman, B. 2000, ApJ, 538, 363

Basri, G., \& Reiners, A. 2006, AJ, 132, 663

Bean, J. L., Seifahrt, A., Hartman, H., Nilsson, H., Wiedemann, G., Reiners, A., Dreizler, S., \& Henry, T. J. 2010, ApJ, 713, 410

Bennett, D., et al. 2008, ApJ, 684, 663

Binney, J., \& Merrifield, M. 1998, Galactic Astronomy (Princeton, NJ: Princeton Univ. Press)

Blake, C. H., Charbonneau, D., White, R., Marley, M. S., \& Saumon, D. 2007, ApJ, 666, 1198

Blake, C. H., Bloom, J. S., Latham, D. W., Szentgyorgyi, A. H., Skrutskie, M. F., Falco, E. E., \& Starr, D. S. 2008a, PASP, 120, 860

Blake, C. H., Charbonneau, D., White, R. J., Torres, G., Marley, M. S., \& Saumon, D. 2008b, ApJ, 678, L125

Boss, A. P. 2006, ApJ, 644, 79

Bouchy, F., et al. 2008, A\&A, 482, L25

Bouy, H., Brandner, W., Martín, E. L., Delfosse, X., Allard, F., \& Basri, G. 2003, AJ, 126, 1526

Bouy, H., et al. 2004, A\&A, 423, 341

Brown, T., Libbrecht, K., \& Charbonneau, D. 2003, PASP, 114, 826

Burgasser, A., \& Blake, C. H. 2009, AJ, 137, 4621

Burgasser, A. J., Liu, M. C., Ireland, M. J., Cruz, K. L., \& Dupuy, T. J. 2008, ApJ, 681,579

Burgasser, A. J., Ried, N. I., Siegler, N., Close, L., Allen, P., Lowrance, P., \& Gizis, J. 2007, in Protostars and Planets, ed. B. Reipurth \& K. Keil (Tucson, AZ: Univ. Arizona Press)

Burrows, A., Hubbard, W. B., Lunine, J. I., \& Liebert, J. 2001, Rev. Mod. Phys., 73,719

Butler, R. P., Marcy, G. W., Williams, E., McCarthy, C., Dosanjh, P., \& Vogt, S. S. 1996, PASP, 108, 500

Caccin, B., Cavallini, F., Ceppatelli, G., Righini, A., \& Sambuco, A. M. 1985, A\&A, 149, 357

Campbell, B. 1983, PASP, 95, 577

Chabrier, G., Baraffe, I., \& Allard, C. 2000, ApJ, 542, 464 
Chabrier, G., Gallardo, J., \& Baraffe, I. 2007, A\&A, 472, 17

Chauvin, G., et al. 2005, A\&A, 438, 25

Claret, A. 2000, A\&A, 363, 1081

Cochran, W. D. 1988, ApJ, 334, 349

Cummings, A., Butler, R. P., Marcy, G. W., Vogt, S. S., Wright, J. T., \& Fischer, D. A. 2008, PASP, 120,531

Cushing, C. C., \& Vacca, W. D. 2006, AJ, 131, 1797

Dahn, C. C., et al. 2002, AJ, 124, 1170

Dahn, C. C., et al. 2008, ApJ, 685, 548

Delfosse, X., Forville, T., Ségransan, D., Beuzit, J.-L., Udry, S., Perrier, C., \& Mayor, M. 2000, A\&A, 364, 217

Dupuy, T., Liu, M. C., \& Bowler, B. P. 2009, ApJ, 706, 328

Endl, M., Cochran, W. D., Kuster, M., Paulson, D. B., Wittenmyer, R. A., MacQueen, P. J., \& Tull, R. G. 2006, ApJ, 649, 436

Erskine, D. J., Edelstein, J., Harbeck, D., \& Lloyd, J. 2005, Proc. SPIE, 5905, 249

Faherty, J. K., Burgasser, A. J., Cruz, K. L., Shara, M. M., Walter, F. M., \& Gelino, C. R. 2009, AJ, 137, 1

Figueira, P., Pepe, F., Lovis, C., \& Mayor, M. 2010a, A\&A, 515, 106

Figueira, P., et al. 2010b, A\&A, 511, 55

Freedman, R., Marley, M., \& Lodders, K. 2008, ApJS, 174, 504

Gelino, C. R., Kulkarni, S. R., \& Stephens, D. C. 2006, PASP, 118, 611

Gizis, J. E., \& Reid, I. N. 2006, ApJ, 575, 484

Gould, A., Dorsher, S., Gaudi, B. S., \& Udalski, A. 2006a, Acta Astron., 56, 1

Gould, A., et al. 2006b, ApJ, 644, 37

Gray, D. F. 1992, The Observation and Analysis of Stellar Photospheres (Cambridge: Cambridge Univ. Press)

Griffin, R., \& Griffin, R. 1973, MNRAS, 162, 243

Hatzes, A. P., \& Cochran, W. D. 1993, ApJ, 413, 339

Hauschildt, P. H., Allard, F., \& Baron, E. 1999, ApJ, 512, 377

Hebb, L., et al. 2010, ApJ, 708, 224

Horne, K. 1986, PASP, 98, 609

Huélamo, N., et al. 2008, A\&A, 489, 9

Ida, S., \& Lin, D. N. C. 2005, ApJ, 626, 1045

Ireland, M. J., Kraus, A., Martinache, F., Lloyd, J. P., \& Tuthill, P. J. 2008, ApJ, 678,463

Joergens, V. 2008, A\&A, 492, 545

Joergens, V., \& Müller, A. 2007, ApJ, 666, L113

Johnson, J. A., Butler, R. P., Marcy, W. G., Fischer, D. A., Vogt, S. S., Wright, J. T., \& Peek, K. M. G. 2007, ApJ, 670, 833

Johnson, R. H., \& Soderblom, D. R. 1987, AJ, 93, 684

Joshi, Y., et al. 2009, MNRAS, 392, 1532

Killic, M., Brown, W. R., Allende Prieot, C., Pinsonneault, M. H., \& Kenyon, S. J. 2007, ApJ, 664, 1088

Konopacky, Q., et al. 2010, ApJ, 711, 1087

Kürster, M., et al. 2003, A\&A, 403, 1077

Laughlin, G., Bodenheimer, P., \& Adams, F. C. 2004, ApJ, 612, 73

Livingston, W., \& Wallace, L. 1991, An Atlas of the Solar Spectrum in the Infrared from 1850 to 9000 1/cm, NSO Technical Report (Tucson, AZ: National Solar Observatory, National Optical Astronomy Observatory)

Lodders, K. 2003, ApJ, 591, 1220

Lucy, L. B. 1974, AJ, 79, 745

Luhman, K. L., Hernandex, J., Downes, J. J., Hartman, L., \& Briceno, C. 2008, ApJ, 688, 362
Luhman, K. L., Joergens, V., Lada, C., Muzerolle, J., Pscucci, I., \& White, R. 2006, in Protostars and Planets V, ed. B. Reipurth, D. Jewitt, \& K. Keil (Tucson, AZ: Univ. Arizona Press)

Mahadevan, S., \& Ge, J. 2009, ApJ, 692, 1590

Markwardt, C. B. 2009, in ASP Conf. Ser. 411, Astronomical Data Analysis Software and Systems XVIII, ed. D. A. Bohlender, D. Durand, \& P. Dowler (San Francisco, CA: ASP), 251

Marley, M. S., Seager, S., Saumon, D., Lodders, K., Ackerman, A. S., Freedman, R. S., \& Fan, X. 2002, ApJ, 568, 335

Martín, E. L., Guenther, E., Zapatero Osorio, M. R., Bouy, H., \& Wainscoat, R 2006, ApJ, 644, 75

Martinache, F., Rojas-Ayala, B., Ireland, M. J., Lloyd, J. P., \& Tuthill, P. G. 2009, ApJ, 695, 1183

Maxted, P. F. L., \& Jeffries, R. D. 2005, MNRAS, 362, 45

Mazeh, T., \& Zucker, S. 1994, Ap\&SS, 212, 349

McLean, I. S., et al. 1998, Proc. SPIE, 3354, 566

Nelder, J. A., \& Mead, R. 1965, Comput. J., 7, 308

Payne, M. J., \& Lodato, G. 2007, MNRAS, 381, 1597

Prato, L., Huerta, M., Johns-Krull, C. M., Mahmud, N., Jaffe, D. T., \& Hartigan, P. 2008, ApJ, 687, 103

Press, W. H., Flannery, B. P., \& Teukolsky, S. A. 1986, Numerical Recipes: The art of Scientific Computing (Cambridge: Cambridge Univ. Press)

Ramsey, L. W., Barnes, J., Redman, S. L., Jones, H. R. A., Wolszczan, A., Bongiorno, S., Engel, L., \& Jenkins, J. 2008, PASP, 120, 887

Ranyer, J. T., Cushing, M. C., \& Vacca, W. D. 2009, ApJS, 185, 289

Reid, N. I., Lewitus, E., Allen, P. R., Cruz, K. L., \& Burgasser, A. J. 2006, AJ, 132,891

Reid, N. I., et al. 2000, AJ, 119, 369

Reiners, A., \& Basri, G. 2006, AJ, 131, 1806

Reiners, A., \& Basri, G. 2008, ApJ, 684, 1390

Reiners, A., \& Basri, G. 2009, ApJ, 705, 1416

Reiners, A., Bean, J. L., Huber, K. F., Dreizler, S., Seifahrt, A., \& Czesla, S. 2010, ApJ, 710, 432

Rothman, L., et al. 2009, J. Quant. Spectrosc. Radiat. Transfer, 110, 533

Salim, S., Lépine, S., Rich, M. R., \& Shara, M. M. 2003, ApJ, 586, 149

Saumon, D., \& Marley, M. S. 2008, ApJ, 689, 1327

Schilbach, E., Röser, S., \& Scholz, R.-D. 2009, A\&A, 493, 27

Seifahrt, A., \& Käufl, H. U. 2008, A\&A, 491, 929

Seifahrt, A., Reiners, A., Almaghrbi, K. A. M., \& Basri, G. 2010, A\&A, 512, 37

Smith, M. A. 1982, ApJ, 253, 727

Stassun, K. G., Mathieu, R. D., \& Valenti, J. A. 2006, Nature, 440, 311

Stumpf, M. B., et al. 2008, A\&A, submitted (arXiv:0811.0556)

Udry, S., et al. 2007, A\&A, 469, 43

Vogel, H. C. 1901, ApJ, 13, 324

Whitworth, A., Bate, M. R., Nordlund, A., Reipurth, Bo., \& Zinnecker, H. 2007, in Protostars and Planets V, ed. B. Reipurth, D. Jewitt, \& K. Keil (Tucson, AZ: Univ. Arizona Press)

Wielen, R. 1977, A\&A, 60, 263

Xia, F., Ren, S., \& Fu, Y. 2008, Ap\&SS, 314, 51

Zapatero Osorio, M. R., Martín, E. L., Béjar, V. J. S., Bouy, H., Deshpande, R., \& Wainscoat, R. J. 2007, ApJ, 666, 1205

Zapatero Osorio, M. R., Martín, E. L., del Burgo, C., Deshpande, R., Rodler, F., \& Montgomery, M. M. 2009, A\&A, 505, 5

Zechmeister, M., Kürster, M., \& Endl, M. 2009, A\&A, 505, 859 